\title{
INFORMATION RANKING AND POWER LAWS ON TREES
}

\author{
PREDRAG R. JELENKOVIĆ* AND \\ MARIANA OLVERA-CRAVIOTO, ${ }^{* *}$ Columbia University
}

\begin{abstract}
In this paper we consider the stochastic analysis of information ranking algorithms of large interconnected data sets, e.g. Google's PageRank algorithm for ranking pages on the World Wide Web. The stochastic formulation of the problem results in an equation of the form $R \stackrel{\mathrm{D}}{=} Q+\sum_{i=1}^{N} C_{i} R_{i}$, where $N, Q,\left\{R_{i}\right\}_{i \geq 1}$, and $\left\{C, C_{i}\right\}_{i \geq 1}$ are independent nonnegative random variables, the $\left\{C, C_{i}\right\}_{i \geq 1}$ are identically distributed, and the $\left\{R_{i}\right\}_{i \geq 1}$ are independent copies of $R$; ' $=$ ' stands for equality in distribution. We study the asymptotic properties of the distribution of $R$ that, in the context of PageRank, represents the frequencies of highly ranked pages. The preceding equation is interesting in its own right since it belongs to a more general class of weighted branching processes that have been found to be useful in the analysis of many other algorithms. Our first main result shows that if $\mathrm{E} N \mathrm{E}\left[C^{\alpha}\right]=1, \alpha>0$, and $Q, N$ satisfy additional moment conditions, then $R$ has a power law distribution of index $\alpha$. This result is obtained using a new approach based on an extension of Goldie's (1991) implicit renewal theorem. Furthermore, when $N$ is regularly varying of index $\alpha>1, \mathrm{E} N \mathrm{E}\left[C^{\alpha}\right]<1$, and $Q, C$ have higher moments than $\alpha$, then the distributions of $R$ and $N$ are tail equivalent. The latter result is derived via a novel sample path large deviation method for recursive random sums. Similarly, we characterize the situation when the distribution of $R$ is determined by the tail of $Q$. The preceding approaches may be of independent interest, as they can be used for analyzing other functionals on trees. We also briefly discuss the engineering implications of our results.
\end{abstract}

Keywords: Information ranking; stochastic recursion; stochastic fixed point equation; weighted branching process; power law; regular variation; implicit renewal theory; large deviation

2010 Mathematics Subject Classification: Primary 60H25

Secondary 60J80; 60F10; 60K05

\section{Introduction}

We consider a problem of ranking large interconnected information (data) sets, e.g. ranking pages on the World Wide Web (Web). A solution to this problem is given by Google's PageRank algorithm, the details of which are presented in Section 1.1. Given the large scale of these information sets, we adopt a stochastic approach to the page ranking problem, e.g. Google's PageRank algorithm. The stochastic formulation naturally results in an equation of the form

$$
R \stackrel{\mathrm{D}}{=} Q+\sum_{i=1}^{N} C_{i} R_{i},
$$

Received 21 October 2009; revision received 16 July 2010.

* Postal address: Department of Electrical Engineering, Columbia University, New York, NY 10027, USA.

** Postal address: Department of Industrial Engineering and Operations Research, Columbia University, New York, NY 10027, USA. Email address: molvera@ieor.columbia.edu 
where $N, Q,\left\{R_{i}\right\}_{i \geq 1}$, and $\left\{C, C_{i}\right\}_{i \geq 1}$ are independent nonnegative random variables, $\mathrm{P}(Q>$ $0)>0$, the $\left\{C, C_{i}\right\}_{i \geq 1}$ are identically distributed, and the $\left\{R_{i}\right\}_{i \geq 1}$ are independent copies of $R$; ' $=$, stands for equality in distribution. We study the asymptotic properties of the distribution of $R$ that, in the context of PageRank, represents the frequencies of highly ranked pages. In somewhat smaller generality, the preceding stochastic setup was first introduced and analyzed in [36] for the PageRank algorithm; the formulation given in (1.1) was later studied in [35].

The canonical representation given by recursion (1.1) is also of independent interest since it belongs to a more general class of weighted branching processes (WBPs) [25], [27], [31]; the connection to WBPs is discussed in more detail in Section 2.3. With a slight abuse of notation, we also refer to our more restrictive processes as WBPs. These processes have been found to be useful in the average-case analysis of many algorithms [32], e.g. the quicksort algorithm [15], and, thus, our study of recursion (1.1) may be useful in these types of applications. Furthermore, when $Q=1$ and $C_{i} \equiv 1$, the steady state solution to (1.1) represents the total number of individuals born in an ordinary branching process. Also, by letting $N$ be a Poisson random variable, and fixing $Q=1$ and $C_{i} \equiv 1,(1.1)$ reduces to the recursion that is satisfied by the busy period of an M/G/1 queue. Similarly, selecting $N=1$ yields the fixed point equation satisfied by the first-order autoregressive process; see Section 2.3 for a more thorough discussion on related processes.

In Section 2 we connect the iterations of recursion (1.1) to an explicit construction of a WBP on a tree, such that the sum of all the weights of the first $n$ generations of the tree are directly related to the $n$th iteration of the recursion. Then, in Section 3 we present explicit estimates for the moments of the total weight, $W_{n}$, of the $n$th generation in the corresponding WBP. Using these moment estimates and the WBP representation, we show in Section 3.1 that, under mild conditions, the iterations of (1.1) converge in distribution to a unique and finite steady state random variable $R$. Hence, under the stated assumptions, this limiting distribution $\mathrm{P}(R \leq x)$ is the unique solution to (1.1). The steady state variable $R$ represents the sum of all the weights in the corresponding branching tree.

Studying the asymptotic tail properties of the constructed steady state solution $R$ to (1.1) represents the main focus of this paper. In particular, we study the possible causes that can result in power tail asymptotics for $\mathrm{P}(R>x)$. We discover that the tail behavior of $R$ can be determined/dominated by the statistical properties of any of the three variables $C, N$, and $Q$. The corresponding results are presented in Sections 4, 5, and 6, respectively. Our emphasis on power law asymptotics is motivated by the well-established empirical fact that the number of pages that point to a specific page (in-degree) on the Web, represented by $N$ in recursion (1.1), follows a power law distribution; other complex data sets, e.g. citations, are found to possess similar power law properties as well.

Our first main result on the tail behavior of $\mathrm{P}(R>x)$ is presented in Theorem 4.2, showing that if $\mathrm{E} N \mathrm{E}\left[C^{\alpha}\right]=1, \alpha>0$, and $Q, N$ satisfy additional moment conditions, then $R$ has a power law distribution of index $\alpha$, with an explicitly characterized constant of proportionality. In particular, when $\alpha$ is an integer, the constant of proportionality of the power law distribution is explicitly computable; see Corollary 4.1. This result is obtained by an extension of Goldie's [16] implicit renewal theorem that we present in Theorem 4.1. This extension may be of independent interest since $R$ and $C$ in the statement of Theorem 4.1 can be any two independent random variables that may satisfy a different recursion. In the context of the broader literature on WBPs, our results are related to the studies in [31] (see Theorem 6) and, more recently, in [2], both of which study recursion (1.1) using stable law methods when $Q$ and $\left\{C_{i}\right\}$ are deterministic 
constants. However, these deterministic assumptions fall outside the scope of this paper; for more details, see the discussion in Section 2.3 and the remarks after Theorem 4.2. Outside of these results, the majority of the work on WBPs considers the homogeneous equation $(Q \equiv 0)$, e.g. in [27] the behavior of the distribution of $R$ was characterized using stable law distributions for $0<\alpha \leq 1$. Also, related results for the homogeneous case $(Q \equiv 0)$ and $\alpha>1$ can be found in Theorem 2.2 of [28] and Proposition 7 of [18]. For additional comments on results related to our Theorem 4.2, see the remarks following its statement. Furthermore, this result may provide a new explanation of why power laws are so commonly found in the distribution of wealth since weighted branching processes appear to be reasonable models for the total wealth of a family tree.

In Section 5 we study the case when $N$ is power law and dominates the tail behavior of $R$. This is the case that more closely relates to the original formulation of PageRank and the structure of the Web graph since the in-degree $N$ is well accepted to be a power law. Our main result in this case, stated in Theorem 5.1, shows that, when $N$ is regularly varying of index $\alpha>1, \mathrm{E} N \mathrm{E}\left[C^{\alpha}\right]<1$, and $Q, C$ have higher moments than $\alpha$, then the distribution of $R$ is tail equivalent to that of $N$. Our approach in deriving this result is based on a new sample path heavy-tailed large deviation method for weighted recursions on trees. The key technical result is given in Proposition 5.1 and provides a uniform bound (in $n$ and $x$ ) on the distribution of the total weight of the $n$th generation $\mathrm{P}\left(W_{n}>x\right)$. We would also like to point out that Proposition 5.1 resembles, to some extent, a classical result by Kesten (see Lemma 7 of [5, p. 149]), which provides a uniform bound for the sum of heavy-tailed (subexponential) random variables. The main difference between the latter result and our uniform bound is that $n$ refers to the depth of the recursion in our case, while in Lemma 7 of [5], $n$ is the number of terms in the sum. This makes the derivation of Proposition 5.1 considerably more complicated, and perhaps implausible, if it were not for the fact that we restrict our attention to regularly varying distributions, as opposed to the general subexponential class.

In Section 6 we investigate a third possible source of heavy tails for $R$, namely that which arises from the innovation, $Q$, being power law; see Theorem 6.1. For $N=1$, this result is consistent with a corresponding result for the first-order autoregressive process in Lemma A.3 of [29]. The proofs of more technical results are postponed to Section 7.

Finally, from a mathematical perspective, we would like to emphasize that our sample path large deviation approach, as well as the extension of the implicit renewal theory, provides a new set of tools that can be of potential use in other applications, as well as in studying a broader class of recursions on trees, e.g. we can readily characterize the asymptotic behavior of the distribution that solves $R=Q+\max _{1 \leq i \leq N} C_{i} R_{i}$. Furthermore, from an engineering perspective, our Theorem 5.1 shows that, for highly ranked pages, the PageRank algorithm basically reflects the popularity vote given by the number of references $N$, implying that overly inflated referencing may be advantageous. A more detailed discussion on the engineering implications of the performance and design of ranking algorithms, e.g. PageRank, can be found at the end of Section 5.

\subsection{Google's algorithm: PageRank}

PageRank is an algorithm trademarked by Google, the Web search engine, to assign to each page a numerical weight that measures its relative importance with respect to other pages. We think of the Web as a very large interconnected graph where nodes correspond to pages. The 
Google trademarked algorithm PageRank defines the page rank as

$$
R\left(p_{i}\right)=\frac{1-d}{n}+d \sum_{p_{j} \in M\left(p_{i}\right)} \frac{R\left(p_{j}\right)}{L\left(p_{j}\right)},
$$

where, using Google's notation, $p_{1}, p_{2}, \ldots, p_{n}$ are the pages under consideration, $M\left(p_{i}\right)$ is the set of pages that link to $p_{i}, L\left(p_{j}\right)$ is the number of outbound links on page $p_{j}, n$ is the total number of pages on the Web, and $d$ is a damping factor, usually $d=0.85$. As noted in the original paper by Brin and Page [11], PageRank 'can be calculated using a simple iterative algorithm, and corresponds to the principal eigenvector of the normalized link matrix of the Web. Also, a PageRank for 26 million web pages can be computed in a few hours on a medium size workstation'. Other link-based ranking algorithms for web pages include the HITS algorithm, developed by Kleinberg [24], and the TrustRank algorithm [17].

While, in principle, the solution to (1.2) reduces to the solution of a large system (possibly billions) of linear equations, we believe that finding page ranks in such a way is unlikely to be insightful. Specifically, if we obtain the principal eigenvector of the normalized link matrix, it is hard to obtain from the solution qualitative insights about the relationship between highly ranked pages and the in-degree/out-degree statistical properties of the graph.

In particular, the division by the out-degree, $L\left(p_{j}\right)$ in (1.2), was meant to decrease the contribution of pages with highly inflated referencing, i.e. those pages that basically point/reference possibly indiscriminately to other documents. However, the stochastic approach (to be described in the following sections) reveals that highly ranked pages are essentially insensitive to the parameters of the out-degree distribution, implying that the PageRank algorithm may not reduce the effects of overly inflated referencing (citations, voting) as originally intended, i.e. it may lead to possibly unjustifiable highly ranked pages. An analytical explanation as to why the tail of the rank distribution is dominated by $N$ was first given in [35] and [36]. More discussions on this topic are provided at the end of Section 5.

A stochastic approach to analyze (1.2) is to consider the recursion

$$
R \stackrel{\mathrm{D}}{=} \gamma+c \sum_{i=1}^{N} \frac{R_{i}}{D_{i}},
$$

where $\gamma, c>0$ are constants, $c \mathrm{E}[1 / D]<1, N$ is a random variable independent of the $R_{i} \mathrm{~s}$ and $D_{i} \mathrm{~s}$, the $D_{i} \mathrm{~s}$ are independent and identically distributed (i.i.d.) random variables satisfying $D_{i} \geq 1$, and the $R_{i}$ s are i.i.d. random variables having the same distribution as $R$. In terms of recursion (1.2), $R$ is the rank of a random page, $N$ corresponds to the in-degree of that node, the $R_{i}$ s are the ranks of the pages pointing to it, and the $D_{i}$ s correspond to the out-degrees of each of these pages. The experimental justification of these independence assumptions can be found in [34]. This stochastic setup was first introduced in [36], where the process resulting from a finite number of iterations of (1.3) was analyzed. More recently, in a follow up paper [35], the more general recursion

$$
R \stackrel{\mathrm{D}}{=} Q+\sum_{i=1}^{N} C_{i} R_{i}
$$

was analyzed via Tauberian theorems for the cases when $N$ or $Q$ dominate. In [35], dependency between $N$ and $Q$ is allowed, but additional moment conditions are imposed. Recall that in the setup considered here $N, Q,\left\{R_{i}\right\}_{i \geq 1}$, and $\left\{C, C_{i}\right\}_{i \geq 1}$ are independent nonnegative random variables, $\mathrm{P}(Q>0)>0$, the $\left\{C, C_{i}\right\}_{i \geq 1}$ are identically distributed, and the $\left\{R_{i}\right\}_{i \geq 1}$ are independent copies of $R$. 


\section{Model description}

As outlined above, we study the sequence of random variables that are obtained by iterating (1.1). Specifically, we consider, for $n \geq 0$,

$$
R_{n+1}^{*}=Q_{n}+\sum_{i=1}^{N_{n}} C_{n, i} R_{n, i}^{*},
$$

where the $\left\{R_{n, i}^{*}\right\}_{i \geq 1}$ are i.i.d. copies of $R_{n}^{*}$ from the previous iteration, and $\left\{N_{n}\right\},\left\{C_{n, i}\right\}$, and $\left\{Q_{n}\right\}$ are mutually independent i.i.d. sequences of random variables, independent of the $\left\{R_{n, i}\right\}$; for $n=0$, the $R_{0, i}^{*}$ are i.i.d. copies of the initial value $R_{0}^{*}$.

In this section we will discuss the weak convergence of $R_{n}^{*}$ to a finite random variable $R$, independently of the initial condition $R_{0}^{*}$. In other words, $R$ is the unique solution to (1.1) under the assumptions of Lemma 3.4. In particular, we will construct a process $R^{(n)}$ on a tree that converges almost surely (a.s.) to $R$. These convergence results may be of practical interest as well since ranking algorithms are implemented recursively. The actual proofs are postponed until Section 3.1.

\subsection{Construction of $\boldsymbol{R}$ on a tree}

To better understand the dynamics of our recursion, we give below a sample path construction of the random variable $R$ on a tree. First we construct a random tree $T$. We use the notation $\varnothing$ to denote the root node of $T$, and $A_{n}, n \geq 0$, to denote the set of all individuals in the $n$th generation of $T, A_{0}=\{\varnothing\}$. Let $Z_{n}$ be the number of individuals in the $n$th generation, that is, $Z_{n}=\left|A_{n}\right|$, where $|\cdot|$ denotes the cardinality of a set; in particular, $Z_{0}=1$. We iteratively construct the tree as follows. Let $N^{(0)}$ be the number of individuals born to the root node $\varnothing$, and let $N^{(0)},\left\{N_{i_{1}, \ldots, i_{n}}^{(n)}\right\}_{n \geq 1}$ be i.i.d. copies of $N$. Define

$$
\begin{aligned}
& A_{1}=\left\{i: 1 \leq i \leq N^{(0)}\right\}, \\
& A_{n}=\left\{\left(i_{1}, i_{2}, \ldots, i_{n}\right):\left(i_{1}, \ldots, i_{n-1}\right) \in A_{n-1}, 1 \leq i_{n} \leq N_{i_{1}, \ldots, i_{n-1}}^{(n-1)}\right\} .
\end{aligned}
$$

Then the number of individuals $Z_{n}=\left|A_{n}\right|, n \geq 1$, in the $n$th generation satisfies the branching recursion

$$
Z_{n}=\sum_{\left(i_{1}, \ldots, i_{n-1}\right) \in A_{n-1}} N_{i_{1}, \ldots, i_{n-1}}^{(n-1)} .
$$

Suppose now that individual $\left(i_{1}, \ldots, i_{n}\right)$ in the tree has a weight $\mathcal{C}_{i_{1}, \ldots, i_{n}}^{(n)}$ defined via the recursion

$$
\mathcal{C}_{i_{1}}^{(1)}=C_{i_{1}}^{(1)}, \quad \mathcal{C}_{i_{1}, \ldots, i_{n}}^{(n)}=C_{i_{1}, \ldots, i_{n-1}, i_{n}}^{(n)} \mathcal{C}_{i_{1}, \ldots, i_{n-1}}^{(n-1)}, \quad n \geq 2,
$$

where $\mathcal{C}^{(0)}=1$ is the weight of the root node and the random variables $\left\{C_{i_{1}, \ldots, i_{n}}^{(n)}: n \geq 0, i_{k} \geq 1\right\}$ are i.i.d. with the same distribution as $C$. Note that $\mathcal{C}_{i_{1}, \ldots, i_{n}}^{(n)}$ is equal to the product of all the weights $C^{(\cdot)}$ along the branch leading to node $\left(i_{1}, \ldots, i_{n}\right)$, as depicted in Figure 1 . Now define the process

$$
W_{n}=\sum_{\left(i_{1}, \ldots, i_{n}\right) \in A_{n}} Q_{i_{1}, \ldots, i_{n}}^{(n)} \mathcal{C}_{i_{1}, \ldots, i_{n}}^{(n)}, \quad n \geq 0,
$$

where $A_{n}$ is the set of all individuals in the $n$th generation and $\left\{Q_{i_{1}, \ldots, i_{n}}^{(n)}\right\}_{n \geq 0}$ is a sequence of i.i.d. random variables, independent of everything else, having the same distribution as $Q$ (see Figure 1).

Observe that when $C^{(\cdot)} \equiv 1$ and $Q^{(\cdot)} \equiv 1, W_{n}$ is equal to the number of individuals in the $n$th generation of the corresponding branching process, and, in particular, $Z_{n}=W_{n}$. Otherwise, $W_{n}$ 


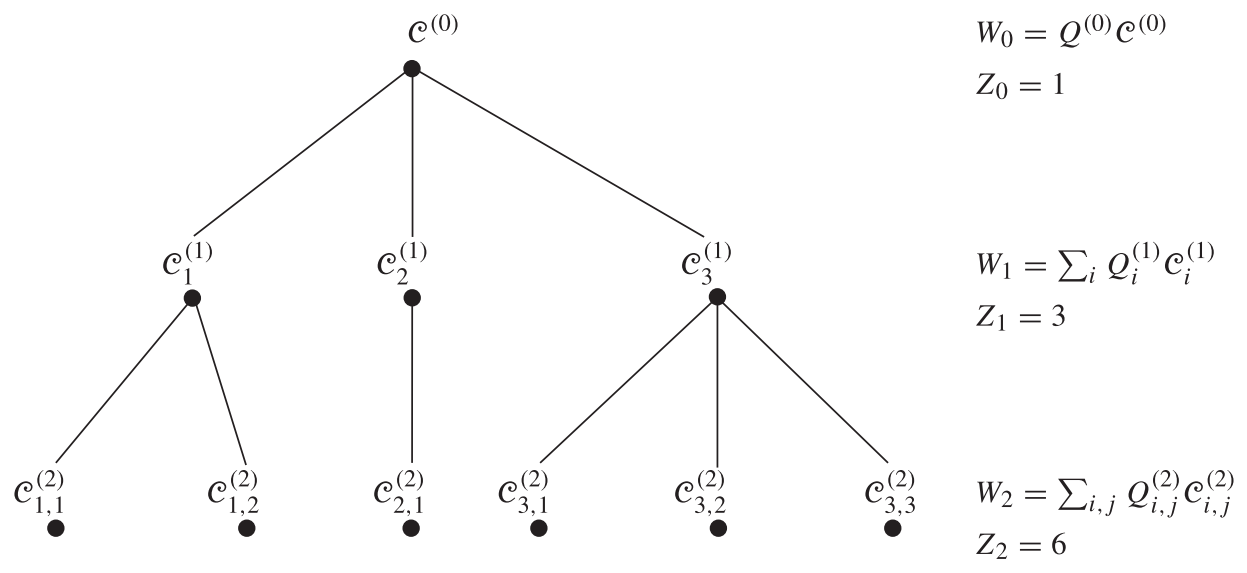

FIgure 1: Construction on a tree.

represents the sum of the weights of all the individuals in the $n$th generation. Related processes known as weighted branching processes have been considered in the existing literature [25], [27], [31] and are discussed in more detail in Section 2.3. With a slight abuse of notation, we also refer to our more restrictive processes as WBPs.

Define the process $\left\{R^{(n)}\right\}_{n \geq 0}$ according to

$$
R^{(n)}=\sum_{k=0}^{n} W_{k}, \quad n \geq 0,
$$

that is, $R^{(n)}$ is the sum of the weights of all the individuals on the tree. Clearly, when $Q . \equiv 1$ and $C^{(\cdot)} \equiv 1, R^{(n)}$ is simply the number of individuals in a branching process up to the $n$th generation. We define the random variable $R$ according to

$$
R:=\lim _{n \rightarrow \infty} R^{(n)}=\sum_{k=0}^{\infty} W_{k} .
$$

Furthermore, it is not hard to see that $R^{(n)}$ satisfies the recursion

$$
R^{(n)}=\sum_{j=1}^{N^{(0)}} C_{j}^{(1)} R_{j}^{(n-1)}+Q^{(0)}
$$

for $n \geq 1$, where the $\left\{R_{j}^{(n-1)}\right\}$ are independent copies of $R^{(n-1)}$ corresponding to the tree starting with individual $j$ in the first generation and ending on the $n$th generation; note that $R_{j}^{(0)}=Q_{j}^{(1)}$.

Moreover, since the tree structure repeats itself after the first generation, $W_{n}$ satisfies

$$
\begin{aligned}
W_{n} & =\sum_{\left(i_{1}, \ldots, i_{n}\right) \in A_{n}} Q_{i_{1}, \ldots, i_{n}}^{(n)} \mathcal{C}_{i_{1}, \ldots, i_{n}}^{(n)} \\
& =\sum_{k=1}^{N^{(0)}} C_{k}^{(1)} \sum_{\left(k, \ldots, i_{n}\right) \in A_{n}} Q_{k, \ldots, i_{n}}^{(n)} \prod_{j=2}^{n} C_{k, \ldots, i_{j}}^{(j)} \\
& \stackrel{\mathrm{D}}{=} \sum_{k=1}^{N} C_{k} W_{n-1, k},
\end{aligned}
$$


where $N, C_{k}$, and $W_{n-1, k}$ are independent of each other and of all other random variables, and $W_{n-1, k}$ has the same distribution as $W_{n-1}$.

\subsection{Connection between $R_{n}^{*}$ and $\boldsymbol{R}^{(n)}$}

We now connect the two processes $R_{n}^{*}$ and $R^{(n)}$, the process obtained by iterating (1.1) and the process obtained from the tree construction, respectively. To do this, define

$$
W_{n}\left(R_{0}^{*}\right)=\sum_{\left(i_{1}, \ldots, i_{n}\right) \in A_{n}} R_{0,\left(i_{1}, \ldots, i_{n}\right)}^{*} \mathfrak{e}_{i_{1}, \ldots, i_{n}}^{(n)},
$$

where the $R_{0,(i)}^{*}$ are i.i.d. copies, independent of everything else, of the initial condition $R_{0}^{*}$, and the weights $\mathcal{C}^{(n)}$ are those defined in Section 2.1. In words, $W_{n}\left(R_{0}\right)$ is the sum of all the weights in the $n$th generation of the tree with the coefficients $Q{ }^{(n)}$ substituted by the corresponding $R_{0,(\cdot)}^{*}$. It can be verified that

$$
R_{n}^{*} \stackrel{\mathrm{D}}{=} R^{(n-1)}+W_{n}\left(R_{0}^{*}\right)
$$

see [20] for additional details. Since $R^{(n-1)} \rightarrow R$ a.s., it follows from Slutsky's theorem (see Theorem 1 of $\left[12\right.$, p. 254]) that if $W_{n}\left(R_{0}^{*}\right) \stackrel{\mathrm{D}}{\rightarrow} 0$ then

$$
R_{n}^{*} \stackrel{\mathrm{D}}{\rightarrow} R
$$

where ' $\stackrel{\mathrm{D}}{\rightarrow}$ ' denotes convergence in distribution. The proof of this convergence and that of the finiteness of $R$ are given in Section 3.1. Understanding the asymptotic properties of the distribution of $R$, as defined by (2.2), is the main objective of this paper.

\subsection{Related processes}

As mentioned earlier, the stochastic equation defined in (1.1) leads to the analysis of a process known in the literature as a weighted branching process (WBP). WBPs were introduced by Rösler [31] in a construction that is more general than ours. More precisely, each individual in the tree has potentially an infinite number of offspring, and each offspring inherits a certain (nonnegative) weight from its parent and multiplies it by a factor $T_{i}$, where the index $i$ refers to its birth order (i.e. a first born multiplies his/her inheritance by $T_{1}$, a second born by $T_{2}$, etc.). Each individual branches independently, using an independent copy of the sequence $T_{1}, T_{2}, \ldots$ However, within the sequence, $T_{1}, T_{2}, \ldots$ can be dependent. Only individuals whose weights are different than 0 are considered to be alive. The construction we give in this paper would correspond to having

$$
T_{i}=C_{i} \mathbf{1}_{\{N \geq i\}} .
$$

The definition of a WBP described above leads to a stochastic recursion for the total weight of the $n$th generation of the form

$$
W_{n} \stackrel{\mathrm{D}}{=} \sum_{i=1}^{\infty} T_{i} W_{n-1, i}
$$

and a corresponding nonhomogeneous fixed point equation of the form

$$
R \stackrel{\mathrm{D}}{=} \sum_{i=1}^{\infty} T_{i} R_{i}+Q .
$$

In the construction given in [31], the $\left\{T_{i}\right\}$ and $Q$ are allowed to be dependent as well.

We now briefly describe some of the existing literature on WBPs, most of which considers the homogeneous equation, i.e. $Q \equiv 0$. The nonhomogeneous equation has only been studied 
for the special case when $Q$ and the $\left\{T_{i}\right\}$ are deterministic constants. In particular, Rösler [31, Theorem 5] analyzed the solutions to (2.5) when $Q$ and the $\left\{T_{i}\right\}$ are nonnegative deterministic constants, which implies that $T_{i} \leq 1$ for all $i$ and $\sum_{i} T_{i}^{\alpha} \log T_{i} \leq 0$ for all $\alpha>0$, falling outside the scope of this paper. More results about the solutions to (2.5) for the case when $Q$ and the $T_{i}$ s are real-valued deterministic constants were derived in [2].

Regarding the homogeneous equation, in [31], the martingale structure of $W_{n} / m^{n}$ ( $m=$ $\mathrm{E}\left[\sum_{i} T_{i}\right]$ ) was used to point out the existence of $W=\lim _{n \rightarrow \infty} W_{n} / m^{n}$, and it was shown that positive stable distributions with $\alpha \in(0,2)$ arise when $\mathrm{E}\left[\sum_{i} T_{i}^{\alpha}\right]=1$ and some additional moment conditions are satisfied. Furthermore, for a detailed analysis of the case when $W$ follows a positive stable distribution $(0<\alpha \leq 1)$, see [27]. The convergence of $W_{n} / m^{n}$ to $W$ was studied in [33], and conditions for $W$ to belong to the domain of attraction of an $\alpha$-stable law $(1<\alpha<2)$ were given in [33], along with an analysis of the rate of convergence. A generalization of the WBP described in [31] to a random environment was given in [25], where necessary and sufficient conditions for $W$ to be nondegenerate were derived. The existence of moments of $W$ was studied in [1]. The power law tail of $W$ for the critical case $\mathrm{E}\left[\sum_{i=1}^{N} C_{i}\right]=1$ and $\alpha>1$ was derived in Theorem 2.2 of [28] and Proposition 7 of [18]. For an even longer list of references to WBPs and related work, see [2] and [25].

From the discussion above, it is clear that the prior literature on WBPs is extensive, but we point out that the more specific structure of our model, given by (1.1), as well as our novel analysis via implicit renewal theory, allows us to characterize the asymptotic power law behavior of the distribution of $R$ for all $\alpha>0$ when the $\left\{C_{i}\right\}$ dominate the tail. In addition, we study the nonhomogeneous equation (2.5), while the preceding work primarily focuses on the homogeneous case (2.4). The case when $N$ dominates the tail, which is important for the page ranking problem, has not been considered until very recently in [35] and [36]. In reference to the latter work, our analysis is based on a new sample path approach, while the studies in [35] and [36] use transforms and Tauberian theorems as well as somewhat different assumptions. We will provide more details on these connections throughout the paper in remarks after the corresponding theorems.

From a different mathematical perspective, our model also constitutes a generalization of several important types of processes. For instance, by setting $N \equiv 1,(2.1)$ reduces to an autoregressive process of order 1 . Also, by letting $N$ be a Poisson random variable and fixing $C_{i} \equiv 1$ and $Q \equiv 1,(1.1)$ becomes the recursion that the number of customers in a busy period of an M/G/1 queue satisfies. Recursion (1.2) and its connection to the busy period when the weights $D_{i}$ are equal to a deterministic constant was exploited in [26].

It is worth noting that probabilistic sample path approaches for the busy period $\left(C_{i} \equiv 1\right.$, $Q \equiv 1$ ) were developed in [7], [19], and [37]; the work in [19] and [37] also relies on the theory of cycle maximum [3]. However, for our more general model (random $C_{i} \mathrm{~s}$ ), it is not clear if there is a tractable way of generalizing this analysis. Instead of pursuing the preceding directions, we develop a direct sample path large deviation analysis for recursive random sums that provides greater generality.

\section{Moments of $W_{n}$}

In this section we provide explicit estimates for the moments of the total weight, $W_{n}$, of the $n$th generation that will be used throughout the paper. In particular, we apply these estimates in Section 3.1 to prove that $R_{n}^{*} \stackrel{\mathrm{D}}{\rightarrow} R$, where $R<\infty$ a.s. Our estimates may be of independent interest due to their explicit nature. 
A simple calculation shows that, provided $\mathrm{E}[N], \mathrm{E}[Q]$, and $\mathrm{E}[C]<\infty, \mathrm{E}\left[W_{n}\right]<\infty$ and is given by

$$
\mathrm{E}\left[W_{n}\right]=\mathrm{E}[N] \mathrm{E}[C] \mathrm{E}\left[W_{n-1}\right]=(\mathrm{E}[N] \mathrm{E}[C])^{n} \mathrm{E}\left[W_{0}\right]=(\mathrm{E}[N] \mathrm{E}[C])^{n} \mathrm{E}[Q] .
$$

We give below upper bounds on the general moments of $W_{n}$.

Throughout the paper, we will use $K$ to denote a large positive constant that may be different in different places, say $K=K / 2, K=K^{2}$, etc.

Lemma 3.1. Suppose that $\mathrm{E}\left[Q^{\beta}\right] \mathrm{E}[N] \mathrm{E}\left[C^{\beta}\right]<\infty$ for $0<\beta \leq 1$. Then

$$
\mathrm{E}\left[W_{n}^{\beta}\right] \leq\left(\mathrm{E}\left[C^{\beta}\right] \mathrm{E}[N]\right)^{n} \mathrm{E}\left[Q^{\beta}\right]
$$

for all $n \geq 0$.

Proof. Simply note that

$$
\mathrm{E}\left[W_{n}^{\beta}\right]=\mathrm{E}\left[\left(\sum_{i=1}^{N} C_{i} W_{n-1, i}\right)^{\beta}\right],
$$

and use the well-known inequality $\left(\sum_{i=1}^{k} y_{i}\right)^{\beta} \leq \sum_{i=1}^{k} y_{i}^{\beta}$ for $0<\beta \leq 1, y_{i} \geq 0$ (see, e.g. Exercise 4.2.1 of [12, p. 102]).

The lemma for moments greater than 1 is given below.

Lemma 3.2. Suppose that $\mathrm{E}\left[Q^{\beta}\right]<\infty, \mathrm{E}\left[N^{\beta}\right]<\infty$, and $\mathrm{E}[N] \max \left\{\mathrm{E}\left[C^{\beta}\right], \mathrm{E}[C]\right\}<1$ for some $\beta>1$. Then, there exists a constant $K_{\beta}>0$ such that

$$
\mathrm{E}\left[W_{n}^{\beta}\right] \leq K_{\beta}\left(\mathrm{E}[N] \max \left\{\mathrm{E}\left[C^{\beta}\right], \mathrm{E}[C]\right\}\right)^{n}
$$

for all $n \geq 0$.

The proof of Lemma 3.2 is given in Section 7.1.

Remarks. (i) Recall that when $C \equiv 1$ and $Q \equiv 1, \mathrm{E}\left[W_{n}^{\beta}\right]$ is the $\beta$-moment of a subcritical branching process $Z_{n}$ and our result reduces to $\mathrm{E}\left[Z_{n}^{\beta}\right] \leq K_{\beta}(\mathrm{E}[N])^{n}$, which is in agreement with the classical results from branching processes, e.g. see Corollary 1 of $[6$, p. 18]. Moreover, from the proof of the integer $\beta$ case (given in Section 7.1), it is clear that $\mathrm{E}\left[W_{n}^{\beta}\right]$ scales as $\rho^{\beta n}$ if $\rho^{\beta}>\rho_{\beta}$ and as $\rho_{\beta}^{n}$ if $\rho^{\beta}<\rho_{\beta}$, where $\rho=\mathrm{E}[N] \mathrm{E}[C]$ and $\rho_{\beta}=\mathrm{E}[N] \mathrm{E}\left[C^{\beta}\right]$. Note that this is not quite the same as our upper bounds, and the reason we choose the geometric term $\left(\rho \vee \rho_{\beta}\right)^{n}$ instead is that it makes the proofs simpler and is sufficient for our purposes. Similar techniques to those used in proving the preceding lemmas can yield, with some additional work, lower bounds for the $\beta$-moments of $W_{n}$, showing that the correct leading term is $\left(\rho^{\beta} \vee \rho_{\beta}\right)^{n}$.

(ii) More technical results dealing with the existence of the $\beta$-moments of $W:=\lim _{n \rightarrow \infty} W_{n} / \rho^{n}$ can be found in [1]. There, necessary and sufficient conditions are given for the finiteness of $\mathrm{E}\left[W^{\beta} L(W)\right]$ when $\beta \geq 1$ and $L(\cdot)$ is slowly varying (see Theorems 1.2 and 1.3). In particular, the approach the authors took was to first normalize the process so that $\rho=\mathrm{E}\left[W_{1}\right]=1$, and then impose a condition that in our case reduces to $\rho_{\beta}=\mathrm{E}[N] \mathrm{E}\left[C^{\beta}\right]<1$, that is, they precluded the situation where $W_{n}^{\beta}$ might scale as $\rho_{\beta}^{n}$ when $\rho^{\beta}<\rho_{\beta}$. An example where $\mathrm{E}\left[W_{n}^{\beta}\right]$ scales as $\rho_{\beta}^{n}$ is when $N \equiv 1$, since then $W_{n}^{\beta} \stackrel{\mathrm{D}}{=} Q^{\beta} \prod_{i=1}^{n} C_{i}^{\beta}$. 
(iii) Furthermore, observe that when $\rho=1$ and $\rho_{\beta}<1$ for $\beta>1$, our proof of the lemma shows that $\lim _{n \rightarrow \infty} \mathrm{E}\left[W_{n}^{\beta}\right]<\infty$, but it does not converge to 0 , which is in agreement with [1]. However, since we study $R$, it is necessary to have $\rho<1$ for the finiteness of $\mathrm{E}\left[R^{\beta}\right]$. Otherwise, if $\rho=1$ and $\rho_{\beta}<1, \beta>1$, then $\mathrm{E}\left[R^{(n)}\right]=n \mathrm{E}[Q]$, which, by monotone convergence and (2.2), implies that $\mathrm{E}[R]=\infty$, and, therefore, by convexity, $\mathrm{E}\left[R^{\beta}\right]=\infty$.

\subsection{Convergence of $\boldsymbol{R}_{\boldsymbol{n}}^{*}$ and finiteness of $\boldsymbol{R}$}

As discussed in Section 2.2, there are two issues regarding the process $R_{n}^{*}$ that remain to be addressed. One is the proof that

$$
R_{n}^{*} \stackrel{\mathrm{D}}{\rightarrow} R=\sum_{k=0}^{\infty} W_{k}
$$

for any initial condition $R_{0}^{*}$; the other issue is the finiteness of $R$. The lemma below shows that $R<\infty$ a.s.

Lemma 3.3. Suppose that $\mathrm{E}\left[Q^{\beta}\right]<\infty, \mathrm{E}\left[N^{\beta}\right]<\infty$, and either $\mathrm{E}[N] \mathrm{E}\left[C^{\beta}\right]<1$ for some $0<\beta<1$ or $\mathrm{E}[N] \max \left\{\mathrm{E}[C], \mathrm{E}\left[C^{\beta}\right]\right\}<1$ for some $\beta \geq 1$. Then, $\mathrm{E}\left[R^{\gamma}\right]<\infty$ for all $0<\gamma \leq \beta$, and, in particular, $R<\infty$ a.s. Moreover, if $\beta \geq 1, R^{(n)} \stackrel{L_{\beta}}{\longrightarrow} R$, where $L_{\beta}$ stands for convergence in the $\left(\mathrm{E}|\cdot|^{\beta}\right)^{1 / \beta}$ norm.

Proof. Let

$$
\eta= \begin{cases}\mathrm{E}[N] \mathrm{E}\left[C^{\beta}\right] & \text { if } \beta<1, \\ \mathrm{E}[N] \max \left\{\mathrm{E}[C], \mathrm{E}\left[C^{\beta}\right]\right\} & \text { if } \beta \geq 1 .\end{cases}
$$

Then, by Lemmas 3.1 and 3.2,

$$
\mathrm{E}\left[W_{n}^{\beta}\right] \leq K \eta^{n}
$$

for some $K>0$. Suppose that $\beta \geq 1$. Then, by monotone convergence and Minkowski's inequality,

$$
\begin{aligned}
\mathrm{E}\left[R^{\beta}\right] & =\mathrm{E}\left[\lim _{n \rightarrow \infty}\left(\sum_{k=0}^{n} W_{k}\right)^{\beta}\right] \\
& =\lim _{n \rightarrow \infty} \mathrm{E}\left[\left(\sum_{k=0}^{n} W_{k}\right)^{\beta}\right] \\
& \leq \lim _{n \rightarrow \infty}\left(\sum_{k=0}^{n} \mathrm{E}\left[W_{k}^{\beta}\right]^{1 / \beta}\right)^{\beta} \\
& \leq K\left(\sum_{k=0}^{\infty} \eta^{k / \beta}\right)^{\beta} \\
& <\infty .
\end{aligned}
$$

This implies that $R<\infty$ a.s. When $0<\beta<1$, use the inequality $\left(\sum_{k=0}^{n} y_{k}\right)^{\beta} \leq \sum_{k=0}^{n} y_{k}^{\beta}$ for any $y_{i} \geq 0$ instead of Minkowski's inequality. Furthermore, for any $0<\gamma \leq \beta$,

$$
\mathrm{E}\left[R^{\gamma}\right]=\mathrm{E}\left[\left(R^{\beta}\right)^{\gamma / \beta}\right] \leq\left(\mathrm{E}\left[R^{\beta}\right]\right)^{\gamma / \beta}<\infty .
$$

That $R^{(n)} \stackrel{L_{\beta}}{\longrightarrow} R$ whenever $\beta \geq 1$ follows from noting that $\mathrm{E}\left[\left|R^{(n)}-R\right|^{\beta}\right]=\mathrm{E}\left[\left(\sum_{k=n+1}^{\infty} W_{k}\right)^{\beta}\right]$ and applying the same arguments used above to obtain the bound $\mathrm{E}\left[\left|R^{(n)}-R\right|^{\beta}\right] \leq K \eta^{n+1}$ $\times\left(1-\eta^{1 / \beta}\right)^{-\beta}$. 
Next, by monotone convergence in (2.3), it can be verified that $R$ must solve

$$
R \stackrel{\mathrm{D}}{=} \sum_{i=1}^{N} C_{i} R_{i}+Q
$$

where the $\left\{R_{i}\right\}_{i \geq 1}$ are i.i.d. copies of $R$, independent of $N, Q$, and $\left\{C_{i}\right\}$.

We now turn our attention to the proof of the convergence of $R_{n}^{*}$ to $R$. Recall from Section 2.2 that

$$
R_{n}^{*} \stackrel{\mathrm{D}}{=} R^{(n-1)}+W_{n}\left(R_{0}^{*}\right)
$$

where

$$
W_{n}\left(R_{0}^{*}\right)=\sum_{\left(i_{1}, \ldots, i_{n}\right) \in A_{n}} R_{0,\left(i_{1}, \ldots, i_{n}\right)}^{*} \mathcal{C}_{i_{1}, \ldots, i_{n}}^{(n)} .
$$

The following lemma shows that $R_{n}^{*} \stackrel{\mathrm{D}}{\rightarrow} R$ for any initial condition $R_{0}^{*}$ satisfying a moment assumption.

Lemma 3.4. For any $R_{0}^{*} \geq 0$, if $\mathrm{E}\left[Q^{\beta}\right]<\infty, \mathrm{E}\left[\left(R_{0}^{*}\right)^{\beta}\right]<\infty$, and $\mathrm{E}[N] \mathrm{E}\left[C^{\beta}\right]<1$ for some $0<\beta \leq 1$, then

$$
R_{n}^{*} \stackrel{\mathrm{D}}{\rightarrow} R
$$

with $\mathrm{E}\left[R^{\beta}\right]<\infty$. Furthermore, under these assumptions, the distribution of $R$ is the unique solution with finite $\beta$-moment to recursion (1.1).

Proof. In view of (3.1), and since $R^{(n)} \rightarrow R$ a.s., the result follows from Slutsky's theorem (see Theorem 1 of $\left[12\right.$, p. 254]) once we show that $W_{n}\left(R_{0}^{*}\right) \stackrel{\mathrm{D}}{\rightarrow} 0$. Recall that $W_{n}\left(R_{0}^{*}\right)$ is the same as $W_{n}$ if we substitute the $Q_{i_{1}, \ldots, i_{n}}$ by the $R_{0,\left(i_{1}, \ldots, i_{n}\right)}^{*}$. Fix $\epsilon>0$. Then

$$
\begin{aligned}
\mathrm{P}\left(W_{n}\left(R_{0}^{*}\right)>\epsilon\right) & \leq \epsilon^{-\beta} \mathrm{E}\left[W_{n}\left(R_{0}^{*}\right)^{\beta}\right] \\
& \leq \epsilon^{-\beta}\left(\mathrm{E}\left[C^{\beta}\right] \mathrm{E}[N]\right)^{n} \mathrm{E}\left[\left(R_{0}^{*}\right)^{\beta}\right] \quad(\text { by Lemma 3.1). }
\end{aligned}
$$

Since, by assumption, the right-hand side converges to 0 as $n \rightarrow \infty$, then $R_{n}^{*} \stackrel{\mathrm{D}}{\rightarrow} R$. Furthermore, $\mathrm{E}\left[R^{\beta}\right]<\infty$ by Lemma 3.3. Clearly, the distribution of $R$ represents the unique solution with finite $\beta$-moment to (1.1), since any other possible solution would have to converge to the same limit.

Remarks. (i) Note that when $\mathrm{E}[N]<1$, the branching tree is a.s. finite and no conditions on the $C$ s are necessary for $R<\infty$ a.s. This corresponds to the second condition in Theorem 1 of [10].

(ii) In view of the same theorem from [10], we could possibly establish the convergence of $R_{n}^{*} \stackrel{\mathrm{D}}{\rightarrow} R<\infty$ under milder conditions. However, since the conditions that we will impose on $N, Q$, and $C$ in the main theorems will be stronger, this lemma is not restrictive. Furthermore, the initial values, $R_{0}^{*}$, are typically small (e.g. constant in applications), and, thus, the polynomial moment condition imposed on $R_{0}^{*}$ is general enough.

\section{The case when the $C$ s dominate: implicit renewal theory}

In this section we study the power law phenomenon that arises from the multiplicative effects of the weights $\left\{C_{i}\right\}$ in (1.1). 


\subsection{Implicit renewal theorem on trees}

One observation that will help gain some intuition about (2.3) is to consider the case when $N \equiv 1$. The process $\left\{R^{(n)}\right\}$ then reduces to a (random coefficient) autoregressive process of order 1 , whose steady state solution satisfies

$$
R \stackrel{\mathrm{D}}{=} Q+C R
$$

where $R$ is independent of $C$ and $Q$. This is precisely one of the stochastic recursions considered in [16] (see also [23]), where it was shown that, under the assumption that $\mathrm{E}\left[C^{\alpha}\right]=1$ and some other technical conditions on the distribution of $C$ and $Q$, we have

$$
\mathrm{P}(R>x) \sim H x^{-\alpha}
$$

for some (computable) constant $H>0$ (see Theorem 4.1 of [16]). The fact that the index of the power law depends on the distribution of the weights is already promising in terms of our goal of identifying other sources of power law behavior.

Informally speaking, the recursions studied in [16] are basically multiplicative away from the boundary. However, (1.1) always has an additive component given by $\sum_{i=1}^{N} C_{i} R_{i}$ regardless of how far from the boundary one may be. Fortunately, due to the heavy-tailed nature of $R$, our intuition says that it is only one of the additive $C_{i} R_{i}$ components that determines the behavior of (1.1); thus, the sum will behave as the maximum term, simplifying to

$$
\mathrm{P}\left(Q+\sum_{i=1}^{N} C_{i} R_{i}>x\right) \sim \mathrm{E}[N] \mathrm{P}(C R>x),
$$

assuming that $Q$ has a light enough tail. This heuristic suggests the following generalization of Theorem 2.3 of [16].

Here, we would like to emphasize that $R$ and $C$ in the following theorem can be any two independent random variables that satisfy the stated conditions, i.e. they do not have to be related by recursion (1.1). Hence, the theorem may be of potential use in other applications. Note that we prove the theorem for a general constant $m$, which in our application refers to $\mathrm{E}[N]$, as suggested by (4.1).

Theorem 4.1. Suppose that $C \geq 0$ a.s., $0<\mathrm{E}\left[C^{\alpha} \log C\right]<\infty$ for some $\alpha>0$, and that the conditional distribution of $\log C$ given $C \neq 0$ is nonarithmetic. Suppose further that $R$ is independent of $C, m \mathrm{E}\left[C^{\alpha}\right]=1$, and that $\mathrm{E}\left[R^{\beta}\right]<\infty$ for any $0<\beta<\alpha$. If

$$
\int_{0}^{\infty}|\mathrm{P}(R>t)-m \mathrm{P}(C R>t)| t^{\alpha-1} \mathrm{~d} t<\infty
$$

then

$$
\mathrm{P}(R>t) \sim H t^{-\alpha}, \quad t \rightarrow \infty
$$

where $H \geq 0$ is given by

$$
H=\frac{1}{m \mathrm{E}\left[C^{\alpha} \log C\right]} \int_{0}^{\infty} v^{\alpha-1}(\mathrm{P}(R>v)-m \mathrm{P}(C R>v)) \mathrm{d} v .
$$

The proof of this theorem follows the same steps as Theorem 2.3 of [16], and is presented in Section 7.2. 
Remarks. (i) As pointed out in [16], the statement of the theorem has content only when $R$ has infinite moment of order $\alpha$, since otherwise the constant $H=\left(\alpha \mathrm{E}[N] \mathrm{E}\left[C^{\alpha} \log C\right]\right)^{-1}$ $\left(\mathrm{E}\left[R^{\alpha}\right]-\mathrm{E}[N] \mathrm{E}\left[(C R)^{\alpha}\right]\right)$ will be 0 by independence of $R$ and $C$.

(ii) Note that some of the assumptions of Theorem 4.1 are different than the corresponding assumptions of Theorem 2.3 of [16]. In particular, it is no longer the case that convexity implies that $\mathrm{E}\left[C^{\alpha} \log C\right]>0$ whenever $\alpha$ solves $m \mathrm{E}\left[C^{\alpha}\right]=1$ and $\mathrm{E}\left[C^{\alpha} \log C\right]<\infty$, since if $m>1$, it is possible to construct counterexamples; hence, the need to include this as an assumption. Another difference is our requirement that $\mathrm{E}\left[R^{\beta}\right]<\infty$ for any $0<\beta<\alpha$. In the case of applying Theorem 4.1 to (1.1), the condition on $\mathrm{E}\left[R^{\beta}\right]$ is not restrictive since we readily obtain the moments of $R$ for $0<\beta<\alpha$ from the computed moments of $W_{n}$ from Section 3 .

(iii) A similar result for the case when $\log C$ is lattice valued can be derived using the corresponding renewal theorem.

In what follows we will use the preceding theorem to derive the asymptotic behavior of $\mathrm{P}(R>x)$, where $R$, as given by (2.2), satisfies recursion (1.1). Here, the main difficulty will be to show that condition (4.2) holds. For brevity, we use $x \vee y$ to denote $\max \{x, y\}$ and $x \wedge y$ to denote $\min \{x, y\}$.

Theorem 4.2. Suppose that $0<\mathrm{E}\left[C^{\alpha} \log C\right]<\infty$ for some $\alpha>0$, that the conditional distribution of $\log C$ given $C \neq 0$ is nonarithmetic, and that $C$ and $R$ are independent, where $R$ is defined by (2.2). Assume that $\mathrm{E}[N] \mathrm{E}\left[C^{\alpha}\right]=1,0<\mathrm{E}\left[Q^{\alpha}\right]<\infty$, and $\mathrm{E}\left[N^{\alpha \vee(1+\epsilon)}\right]<\infty$ for some $0<\epsilon<1$; if $\alpha>1$, assume further that $\mathrm{E}[N] \mathrm{E}[C]<1$. Then

$$
\mathrm{P}(R>t) \sim H t^{-\alpha}, \quad t \rightarrow \infty
$$

where

$$
\begin{aligned}
H & =\frac{1}{\mathrm{E}[N] \mathrm{E}\left[C^{\alpha} \log C\right]} \int_{0}^{\infty} v^{\alpha-1}(\mathrm{P}(R>v)-\mathrm{E}[N] \mathrm{P}(C R>v)) \mathrm{d} v \\
& =\frac{\mathrm{E}\left[\left(\sum_{i=1}^{N} C_{i} R_{i}+Q\right)^{\alpha}-\sum_{i=1}^{N}\left(C_{i} R_{i}\right)^{\alpha}\right]}{\alpha \mathrm{E}[N] \mathrm{E}\left[C^{\alpha} \log C\right]} .
\end{aligned}
$$

Remarks. (i) Note that the second expression for $H$ is more suitable for actually computing it, especially in the case of $\alpha$ being an integer, as will be stated in the forthcoming corollary.

(ii) When $\alpha$ is not an integer, we can derive an explicit bound on $H$ by using the forthcoming Lemma 4.3 and (4.4).

(iii) For the homogeneous equation $(Q \equiv 0)$ and $\alpha>1$, closely related results to our theorem can be found in Theorem 2.2 of [28] and Proposition 7 of [18]. The approach in [28] transforms the recursion $W \stackrel{\mathrm{D}}{=} \sum_{i=1}^{N} C_{i} W_{i}$ for the critical case, $\mathrm{E}[W]=1$ and $\mathrm{E}\left[\sum_{i=1}^{N} C_{i}\right]=1$, to a first-order difference (autoregressive) equation on a different probability space; see Lemma 4.1 of [28]. Note that the tail behavior of $W$ does not imply that of $R$. Furthermore, it appears that the method in [28] does not extend to the nonhomogeneous case since the proof of Lemma 4.1 of [28] critically depends on having both $\mathrm{E}[W]=1$ and $\mathrm{E}\left[\sum_{i=1}^{N} C_{i}\right]=1$, which is only possible when $Q \equiv 0$. For $0<\alpha \leq 1$, the homogeneous equation was studied in [27] using stable laws.

(iv) Related results for the nonhomogeneous equation with deterministic constants $Q,\left\{C_{i}\right\}$, and $N=\infty$, have been considered in [31] (see Theorem 5), and more recently in [2], also using stable laws. 
(v) Moreover, the results obtained in the references cited above appear to be less explicit in the expression for $H$ than the statement of Theorem 4.2, as Corollary 4.1 below illustrates.

(vi) Furthermore, Theorem 4.1 and the preceding technique of Theorem 4.2 can be adapted to analyze other, possibly nonlinear, recursions on trees, e.g. we can characterize the asymptotic behavior of $\mathrm{P}(R>x)$ that solves

$$
R=Q+\max _{1 \leq i \leq N} C_{i} R_{i}
$$

We also want to point out that we can obtain the logarithmic asymptotics of $R$, that is, the behavior of $\log \mathrm{P}(R>x)$, much easier and under less restrictive conditions, e.g. $\log C_{i}$ needs not be nonarithmetic (this condition is required because of the use of the renewal theorem). An upper bound can be obtained from Lemma 3.3 and Markov's inequality. For the lower bound, using the notation from Section 2.1, we obtain

$$
\begin{aligned}
\mathrm{P}(R>x) & \geq \mathrm{P}\left(W_{n}>x\right) \\
& \geq \mathrm{P}\left(\max _{1 \leq i \leq N} C_{i} W_{n-1, i}>x\right) \\
& =\mathrm{E}\left[\left(1-\mathrm{P}\left(C W_{n-1} \leq x\right)^{N}\right)\right] \\
& \geq \mathrm{E}\left[N \mathrm{P}\left(C W_{n-1} \leq x\right)^{N}\right] \mathrm{P}\left(C W_{n-1}>x\right),
\end{aligned}
$$

where in the last step we used the relation $1-t^{m} \geq m t^{m}(1-t)$ for $0 \leq t \leq 1$. Now we use the fact that $\mathrm{P}\left(C W_{n-1} \leq x\right) \geq \mathrm{P}(R \leq x)$ for all $x$ to show that

$$
\begin{aligned}
\mathrm{P}(R>x) & \geq \mathrm{E}\left[N \mathrm{P}(R \leq x)^{N}\right] \mathrm{P}\left(C W_{n-1}>x\right) \\
& \geq \mathrm{E}\left[N \mathrm{P}(R \leq x)^{N}\right] \mathrm{P}\left(C_{1} \max _{1 \leq i \leq N} C_{2, i} W_{n-2, i}>x\right) \\
& \geq \mathrm{E}\left[N \mathrm{P}(R \leq x)^{N}\right] \mathrm{E}\left[N \mathrm{P}\left(C_{1} C_{2} W_{n-2} \leq x\right)^{N}\right] \mathrm{P}\left(C_{1} C_{2} W_{n-2}>x\right),
\end{aligned}
$$

which, by using $\mathrm{P}\left(C_{1} C_{2} W_{n-2} \leq x\right) \geq \mathrm{P}(R \leq x)$ for all $x$, yields

$$
\mathrm{P}(R>x) \geq\left(\mathrm{E}\left[N \mathrm{P}(R \leq x)^{N}\right]\right)^{2} \mathrm{P}\left(C_{1} C_{2} W_{n-2}>x\right) .
$$

Next, by continuing this inductive argument we obtain

$$
\mathrm{P}(R>x) \geq\left(\mathrm{E}\left[N \mathrm{P}(R \leq x)^{N}\right]\right)^{n} \mathrm{P}\left(Q \prod_{i=1}^{n} C_{i}>x\right) .
$$

Finally, for any $0<\epsilon<1$, we can choose $x_{0}$ such that $\mathrm{E}\left[N \mathrm{P}\left(R \leq x_{0}\right)^{N}\right] \geq(1-\epsilon) \mathrm{E}[N]$, implying that, for all $n \geq 0$ and $x \geq x_{0}$,

$$
\begin{aligned}
\mathrm{P}(R>x) & \geq(1-\epsilon)^{n}(\mathrm{E}[N])^{n} \mathrm{P}\left(Q \prod_{i=1}^{n} C_{i}>x\right) \\
& \geq \mathrm{P}\left(Q>\frac{1}{\log x}\right)(1-\epsilon)^{n}(\mathrm{E}[N])^{n} \mathrm{P}\left(\prod_{i=1}^{n} C_{i}>x \log x\right) .
\end{aligned}
$$

Now define $S_{n}=\log C_{1}+\cdots+\log C_{n}, \kappa(\theta)=\log \mathrm{E}\left[C^{\theta}\right]$, and choose $\alpha$ to be the solution to $\kappa(\alpha)=-\log \mathrm{E}[N]$ (i.e. $\mathrm{E}[N] \mathrm{E}\left[C^{\alpha}\right]=1$ ). The logarithmic asymptotics can be obtained by 
choosing $n=n(x)=\log (x \log x) / \mu_{\alpha}$, where $\mu_{\alpha}=\kappa^{\prime}(\alpha)=\mathrm{E}\left[C^{\alpha} \log C\right] / \mathrm{E}\left[C^{\alpha}\right]>\kappa^{\prime}(0)=$ $\mathrm{E}[\log C]$ by convexity of $\kappa(\cdot)$. Then, by Theorem 2.1 of [4, Chapter XIII],

$$
\begin{aligned}
\liminf _{x \rightarrow \infty} \frac{\log \mathrm{P}(R>x)}{\log x} & \geq \frac{\log ((1-\epsilon) \mathrm{E}[N])}{\mu_{\alpha}}+\liminf _{x \rightarrow \infty} \frac{\log \mathrm{P}\left(S_{n(x)}>\mu_{\alpha} n(x)\right)}{\mu_{\alpha} n(x)} \\
& =\frac{\log (1-\epsilon)}{\mu_{\alpha}}-\alpha .
\end{aligned}
$$

Hence, we can derive with a considerably smaller effort the following theorem.

Theorem 4.3. Suppose that $0<\mathrm{E}\left[C^{\alpha} \log C\right]<\infty$ for some $\alpha>0$, and that $R$ is given by (2.2). Assume that $\mathrm{E}[N] \mathrm{E}\left[C^{\alpha}\right]=1,0<\mathrm{E}\left[Q^{\alpha}\right] \mathrm{E}\left[N^{\alpha}\right]<\infty$; if $\alpha>1$, assume further that $\mathrm{E}[N] \mathrm{E}[C]<1$. Then

$$
\log \mathrm{P}(R>t) \sim-\alpha \log t, \quad t \rightarrow \infty .
$$

Therefore, the majority of the work in proving Theorem 4.2 goes into the derivation of the exact asymptotic. Furthermore, it is worth noting that the logarithmic approach, although less precise, can be obtained in a more general setting. For example, we can have $C^{(\cdot)}$ to be dependent across different generations, as in the so-called WBP in a random environment. Here, we could derive the asymptotics of $\log \mathrm{P}(R>x)$ if $\mathrm{E}\left[\left(\prod_{i=1}^{n} C_{(1,1, \ldots, 1)}^{(n)}\right)^{\alpha}\right]$ satisfies the polynomial-type Gärtner-Ellis conditions that were recently considered in [21].

Corollary 4.1. For an integer $\alpha \geq 1$, and under the same assumptions as Theorem 4.2, the constant $H$ can be explicitly computed as a function of $\mathrm{E}\left[R^{k}\right], \mathrm{E}\left[C^{k}\right]$, and $\mathrm{E}\left[Q^{k}\right], 0 \leq k \leq$ $\alpha-1$. In particular, for $\alpha=1$,

$$
H=\frac{\mathrm{E}[Q]}{\mathrm{E}[N] \mathrm{E}[C \log C]},
$$

and, for $\alpha=2$,

$$
\begin{gathered}
H=\frac{\mathrm{E}\left[Q^{2}\right]+2 \mathrm{E}[Q] \mathrm{E}[C] \mathrm{E}[N] \mathrm{E}[R]+\mathrm{E}[N(N-1)](\mathrm{E}[C] \mathrm{E}[R])^{2}}{2 \mathrm{E}[N] \mathrm{E}\left[C^{2} \log C\right]}, \\
\mathrm{E}[R]=\frac{\mathrm{E}[Q]}{1-\mathrm{E}[N] \mathrm{E}[C]} .
\end{gathered}
$$

Proof. The proof follows directly from multinomial expansions of the second expression for $H$ in Theorem 4.2.

Before giving the proof of Theorem 4.2 we state the following three preliminary lemmas. The proof of Lemma 4.2 is given in Section 7.1 and the proof of Lemma 4.3 is given in Section 7.2.

Lemma 4.1. Suppose that $0<\mathrm{E}\left[C^{\alpha} \log C\right]<\infty$ for some $\alpha>0$ and $\mathrm{E}[N] \mathrm{E}\left[C^{\alpha}\right]=1$; if $\alpha>1$, suppose further that $\mathrm{E}[N] \mathrm{E}[C]<1$. Assume also that $\mathrm{E}\left[Q^{\alpha}\right]<\infty$ and $\mathrm{E}\left[N^{\alpha \vee 1}\right]<\infty$. Then

$$
\mathrm{E}\left[R^{\beta}\right]<\infty
$$

for all $0<\beta<\alpha$.

Proof. The derivative condition $0<\mathrm{E}\left[C^{\alpha} \log C\right]<\infty$ and $\mathrm{E}[N] \mathrm{E}\left[C^{\alpha}\right]=1$ imply that $\mathrm{E}[N] \mathrm{E}\left[C^{\beta}\right]<1$ for all $\beta<\alpha$ that are sufficiently close to $\alpha$. Hence, the conclusion of the result follows from Lemma 3.3. 
Let $\lceil x\rceil$ be the smallest integer greater than or equal to $x$.

Lemma 4.2. Let $\beta>1$, and let $p=\lceil\beta\rceil \in\{2,3,4, \ldots\}$. For any sequence of nonnegative i.i.d. random variables $\left\{Y, Y_{i}\right\}_{i \geq 1}$ and any $k \in\{1,2,3, \ldots\}$, we have

$$
\mathrm{E}\left[\left(\sum_{i=1}^{k} Y_{i}\right)^{\beta}-\sum_{i=1}^{k} Y_{i}^{\beta}\right] \leq k^{\beta} \mathrm{E}\left[Y^{p-1}\right]^{\beta /(p-1)} .
$$

Lemma 4.3. Suppose that $\left\{C, C_{i}\right\}$ and $\left\{R, R_{i}\right\}$ are i.i.d. sequences of nonnegative random variables independent of each other and of $N$. Assume that $\mathrm{E}\left[C^{\alpha}\right]<\infty, \mathrm{E}\left[N^{1+\epsilon}\right]<\infty$ for some $0<\epsilon<1$, and $\mathrm{E}\left[R^{\beta}\right]<\infty$ for any $0<\beta<\alpha$. Then

$$
\begin{aligned}
0 & \leq \int_{0}^{\infty}\left(\mathrm{E}[N] \mathrm{P}(C R>t)-\mathrm{P}\left(\max _{1 \leq i \leq N} C_{i} R_{i}>t\right)\right) t^{\alpha-1} \mathrm{~d} t \\
& =\frac{1}{\alpha} \mathrm{E}\left[\sum_{i=1}^{N}\left(C_{i} R_{i}\right)^{\alpha}-\left(\max _{1 \leq i \leq N} C_{i} R_{i}\right)^{\alpha}\right] \\
& <\infty
\end{aligned}
$$

Proof of Theorem 4.2. By Lemma 4.1 we know that $\mathrm{E}\left[R^{\beta}\right]<\infty$ for any $0<\beta<\alpha$. The statement of the theorem with the first expression for $H$ will follow from Theorem 4.1 once we prove condition (4.2) for $m=\mathrm{E}[N]$. Define

$$
R^{*}=\sum_{i=1}^{N} C_{i} R_{i}+Q
$$

Then

$$
\begin{aligned}
|\mathrm{P}(R>t)-\mathrm{E}[N] \mathrm{P}(C R>t)| \leq & \left|\mathrm{P}(R>t)-\mathrm{P}\left(\max _{1 \leq i \leq N} C_{i} R_{i}>t\right)\right| \\
& +\left|\mathrm{P}\left(\max _{1 \leq i \leq N} C_{i} R_{i}>t\right)-\mathrm{E}[N] \mathrm{P}(C R>t)\right| .
\end{aligned}
$$

Since $R \stackrel{\mathrm{D}}{=} R^{*} \geq \max _{1 \leq i \leq N} C_{i} R_{i}$, the first absolute value disappears. For the second absolute value, note that, by the union bound,

$$
\mathrm{E}[N] \mathrm{P}(C R>t)-\mathrm{P}\left(\max _{1 \leq i \leq N} C_{i} R_{i}>t\right)=\mathrm{E}\left[N \mathrm{P}(C R>t)-1+\mathrm{P}(C R \leq t)^{N}\right] \geq 0 .
$$

It follows that

$$
\begin{aligned}
|\mathrm{P}(R>t)-\mathrm{E}[N] \mathrm{P}(C R>t)| \leq & \mathrm{P}(R>t)-\mathrm{P}\left(\max _{1 \leq i \leq N} C_{i} R_{i}>t\right) \\
& +\mathrm{E}[N] \mathrm{P}(C R>t)-\mathrm{P}\left(\max _{1 \leq i \leq N} C_{i} R_{i}>t\right) .
\end{aligned}
$$

Note that we need to only verify that

$$
\int_{0}^{\infty}\left(\mathrm{P}(R>t)-\mathrm{P}\left(\max _{1 \leq i \leq N} C_{i} R_{i}>t\right)\right) t^{\alpha-1} \mathrm{~d} t<\infty,
$$


since

$$
\int_{0}^{\infty}\left(\mathrm{E}[N] \mathrm{P}(C R>t)-\mathrm{P}\left(\max _{1 \leq i \leq N} C_{i} R_{i}>t\right)\right) t^{\alpha-1} \mathrm{~d} t<\infty
$$

by Lemma 4.3. To see this, note that $R \stackrel{\mathrm{D}}{=} R^{*}$ and $\mathbf{1}_{\left\{R^{*}>t\right\}}-\mathbf{1}_{\left\{\max _{1 \leq i \leq N} C_{i} R_{i}>t\right\}} \geq 0$; thus, by Fubini's theorem we have

$$
\int_{0}^{\infty}\left(\mathrm{P}(R>t)-\mathrm{P}\left(\max _{1 \leq i \leq N} C_{i} R_{i}>t\right)\right) t^{\alpha-1} \mathrm{~d} t=\frac{1}{\alpha} \mathrm{E}\left[\left(R^{*}\right)^{\alpha}-\left(\max _{1 \leq i \leq N} C_{i} R_{i}\right)^{\alpha}\right] .
$$

If $0<\alpha \leq 1$, we apply the inequality $\left(\sum_{i=1}^{k} x_{i}\right)^{\beta} \leq \sum_{i=1}^{k} x_{i}^{\beta}$ for $0<\beta \leq 1$ and $x_{i} \geq 0$, to obtain

$$
\mathrm{E}\left[\left(R^{*}\right)^{\alpha}-\left(\max _{1 \leq i \leq N} C_{i} R_{i}\right)^{\alpha}\right] \leq \mathrm{E}\left[Q^{\alpha}+\sum_{i=1}^{N}\left(C_{i} R_{i}\right)^{\alpha}-\left(\max _{1 \leq i \leq N} C_{i} R_{i}\right)^{\alpha}\right],
$$

which is finite by Lemma 4.3 and the assumption that $\mathrm{E}\left[Q^{\alpha}\right]<\infty$. If $\alpha>1$, we use the well-known inequality $\left(\sum_{i=1}^{k} x_{i}\right)^{\alpha} \geq \sum_{i=1}^{k} x_{i}^{\alpha}, x_{i} \geq 0$ (see Exercise 4.2 .1 of [12, p. 102]), to split the expectation as

$$
\begin{aligned}
\mathrm{E}\left[\left(R^{*}\right)^{\alpha}-\left(\max _{1 \leq i \leq N} C_{i} R_{i}\right)^{\alpha}\right]= & \mathrm{E}\left[\left(R^{*}\right)^{\alpha}-\sum_{i=1}^{N}\left(C_{i} R_{i}\right)^{\alpha}\right] \\
& +\mathrm{E}\left[\sum_{i=1}^{N}\left(C_{i} R_{i}\right)^{\alpha}-\left(\max _{1 \leq i \leq N} C_{i} R_{i}\right)^{\alpha}\right]
\end{aligned}
$$

which can be done since both expressions inside the expectations on the right-hand side are nonnegative. The second expectation is again finite by Lemma 4.3. To see that the first expectation is finite, let $S=\sum_{i=1}^{N} C_{i} R_{i}$ and note that $R^{*}=S+Q$, where $S$ and $Q$ are independent. Let $p=\lceil\alpha\rceil$, and note that $1 \leq p-1<\alpha$. Then, by Lemma 4.2,

$$
\begin{aligned}
\mathrm{E}\left[\left(R^{*}\right)^{\alpha}-\sum_{i=1}^{N}\left(C_{i} R_{i}\right)^{\alpha}\right] & =\mathrm{E}\left[(S+Q)^{\alpha}-S^{\alpha}\right]+\mathrm{E}\left[\left(\sum_{i=1}^{N} C_{i} R_{i}\right)^{\alpha}-\sum_{i=1}^{N}\left(C_{i} R_{i}\right)^{\alpha}\right] \\
& \leq \mathrm{E}\left[(S+Q)^{\alpha}-S^{\alpha}\right]+\mathrm{E}\left[N^{\alpha}\right]\left(\mathrm{E}\left[(C R)^{p-1}\right]\right)^{\alpha /(p-1)} .
\end{aligned}
$$

The second expectation is finite since, by Lemma 3.3, $\mathrm{E}\left[R^{\beta}\right]<\infty$ for any $0<\beta<\alpha$. For the first expectation, we use the inequality

$$
(x+t)^{\kappa} \leq \begin{cases}x^{\kappa}+t^{\kappa}, & 0<\kappa \leq 1, \\ x^{\kappa}+\kappa(x+t)^{\kappa-1} t, & \kappa>1\end{cases}
$$

for any $x, t \geq 0$. We apply the second expression $p-1$ times and then the first expression to obtain

$$
\begin{aligned}
(x+t)^{\alpha} & \leq x^{\alpha}+\alpha(x+t)^{\alpha-1} t \\
& \leq \cdots \\
& \leq x^{\alpha}+\sum_{i=1}^{p-2} \alpha^{i} x^{\alpha-i} t^{i}+\alpha^{p-1}(x+t)^{\alpha-p+1} t^{p-1} \\
& \leq x^{\alpha}+\alpha^{p} t^{\alpha}+\alpha^{p} \sum_{i=1}^{p-1} x^{\alpha-i} t^{i}
\end{aligned}
$$


We conclude that

$$
\mathrm{E}\left[(S+Q)^{\alpha}-S^{\alpha}\right] \leq \alpha^{p} \mathrm{E}\left[Q^{\alpha}\right]+\alpha^{p} \sum_{i=1}^{p-1} \mathrm{E}\left[S^{\alpha-i}\right] \mathrm{E}\left[Q^{i}\right],
$$

where $\mathrm{E}\left[S^{\alpha-i}\right] \leq \mathrm{E}\left[\left(R^{*}\right)^{\alpha-i}\right]<\infty$ for any $1 \leq i \leq p-1$ by Lemma 3.3.

Finally, applying Theorem 4.1 gives

$$
\mathrm{P}(R>t) \sim H t^{-\alpha}
$$

where $H=\left(\mathrm{E}[N] \mathrm{E}\left[C^{\alpha} \log C\right]\right)^{-1} \int_{0}^{\infty} v^{\alpha-1}(\mathrm{P}(R>v)-\mathrm{E}[N] \mathrm{P}(C R>v)) \mathrm{d} v$.

The second expression for $H$ follows by Fubini's theorem, similarly as we have done in (4.3).

\section{The case when $N$ dominates}

We now turn our attention to the distributional properties of $R^{(n)}$ and $R$ when $N$ has a heavytailed distribution (in particular, regularly varying) that is heavier than the potential power law effect arising from the multiplicative weights $\left\{C_{i}\right\}$. This case is particularly important for understanding the behavior of Google's PageRank algorithm since the $C_{i}$ s are smaller than 1 and the in-degree distribution of the Web graph is well accepted to be a power law. We start this section by stating the corresponding lemma that describes the asymptotic behavior of $R^{(n)}$. The main technical difficulty of extending this lemma to steady state $\left(R=R^{(\infty)}\right)$ is to develop a uniform bound for $R-R^{(n)}$, which is enabled by our main technical result of this section, Proposition 5.1. The proofs of the following lemmas follow from a standard application of Breiman's theorem and asymptotics of random power law sums as those found in Lemmas 3.7 and 4.2 of [22]; see [20] for details.

Before stating the lemmas, let us recall that a function $L:[0, \infty) \rightarrow(0, \infty)$ is slowly varying if $L(\lambda x) / L(x) \rightarrow 1$ as $x \rightarrow \infty$ for any $\lambda>0$. We then say that the function $x^{-\alpha} L(x)$ is regularly varying with index $\alpha$.

Lemma 5.1. Suppose that $\mathrm{P}(N>x)=x^{-\alpha} L(x)$ with $L(\cdot)$ slowly varying, $\alpha>1$, and $\mathrm{E}\left[Q^{\alpha+\epsilon}\right]<\infty, \mathrm{E}\left[C^{\alpha+\epsilon}\right]<\infty$ for some $\epsilon>0$. Let $\rho=\mathrm{E}[N] \mathrm{E}[C]$ and $\rho_{\alpha}=\mathrm{E}[N] \mathrm{E}\left[C^{\alpha}\right]$. Then, for any fixed $n \in\{1,2,3, \ldots\}$,

$$
\mathrm{P}\left(R^{(n)}>x\right) \sim \frac{(\mathrm{E}[C] \mathrm{E}[Q])^{\alpha}}{(1-\rho)^{\alpha}} \sum_{k=0}^{n} \rho_{\alpha}^{k}\left(1-\rho^{n-k}\right)^{\alpha} \mathrm{P}(N>x)
$$

as $x \rightarrow \infty$, where $R^{(n)}$ was defined in Section 2.1 .

Lemma 5.2. Suppose that $\mathrm{P}(N>x)=x^{-\alpha} L(x)$ with $L(\cdot)$ slowly varying, $\alpha>1$, and $\mathrm{E}\left[Q^{\alpha+\epsilon}\right]<\infty, \mathrm{E}\left[C^{\alpha+\epsilon}\right]<\infty$ for some $\epsilon>0$. Let $\rho=\mathrm{E}[N] \mathrm{E}[C]$ and $\rho_{\alpha}=\mathrm{E}[N] \mathrm{E}\left[C^{\alpha}\right]$. Then, for any fixed $n \in\{1,2,3, \ldots\}$,

$$
\mathrm{P}\left(W_{n}>x\right) \sim(\mathrm{E}[C] \mathrm{E}[Q])^{\alpha} \sum_{k=0}^{n-1} \rho_{\alpha}^{k} \rho^{(n-1-k) \alpha} \mathrm{P}(N>x)
$$

as $x \rightarrow \infty$, where $W_{n}$ was defined in Section 2.1. 
From this result, provided $\rho \vee \rho_{\alpha}<1$, it is to be expected that a bound of the form

$$
\mathrm{P}\left(W_{n}>x\right) \leq K \eta^{n} \mathrm{P}(N>x)
$$

might hold for all $n$ and $x \geq 1$, for some $\rho \vee \rho_{\alpha}<\eta<1$. Such a bound will provide the necessary tools to ensure that $R-R^{(n)}$ is negligible for large enough $n$, allowing the exchange of limits in Lemma 5.1. Proving this result is the main technical contribution of this section; the actual proof is given in Section 7.3. This bound may be of independent interest for computing the distributional properties of other recursions on branching trees, e.g. it is straightforward to apply our method to study the solution to

$$
R=Q+\max _{1 \leq i \leq N} C_{i} R_{i}
$$

and similar recursions.

Proposition 5.1. Suppose that $\mathrm{P}(N>x)=x^{-\alpha} L(x)$ with $L(\cdot)$ slowly varying, $\alpha>1$, and $\mathrm{E}\left[C^{\alpha+v}\right]<\infty, \mathrm{E}\left[Q^{\alpha+v}\right]<\infty$ for some $v>0$, and let $\mathrm{E}[N] \max \left\{\mathrm{E}\left[C^{\alpha}\right], \mathrm{E}[C]\right\}<\eta<1$. Then, there exists a constant $K=K(\eta, v)>0$ such that, for all $n \geq 1$ and all $x \geq 1$,

$$
\mathrm{P}\left(W_{n}>x\right) \leq K \eta^{n} \mathrm{P}(N>x) .
$$

We would also like to point out that a bound of type (5.2) resembles a classical result by Kesten (see Lemma 7 of [5, p. 149]) stating that the sum of heavy-tailed (subexponential) random variables satisfies

$$
\mathrm{P}\left(X_{1}+\cdots+X_{n}>x\right) \leq K(1+\epsilon)^{n} \mathrm{P}\left(X_{1}>x\right)
$$

uniformly for all $n$ and $x$, for any $\epsilon>0$ (see also [14] for more recent work). The main difference between this result and (5.2) is that while $n$ above refers to the number of terms in the sum, in (5.2) it refers to the depth of the recursion. This makes the derivation of (5.2) considerably more complicated, and perhaps implausible if it were not for the fact that we restrict our attention to regularly varying distributions, as opposed to the general subexponential class.

In view of (5.2), we can now prove the main theorem of this section.

Theorem 5.1. Suppose that $\mathrm{P}(N>x)=x^{-\alpha} L(x)$ with $L(\cdot)$ slowly varying and $\alpha>1$. Let $\rho=\mathrm{E}[N] \mathrm{E}[C]$ and $\rho_{\alpha}=\mathrm{E}[N] \mathrm{E}\left[C^{\alpha}\right]$. Assume that $\rho \vee \rho_{\alpha}<1$, and that $\mathrm{E}\left[C^{\alpha+\epsilon}\right]<\infty$ and $\mathrm{E}\left[Q^{\alpha+\epsilon}\right]<\infty$ for some $\epsilon>0$. Then,

$$
\mathrm{P}(R>x) \sim \frac{(\mathrm{E}[C] \mathrm{E}[Q])^{\alpha}}{(1-\rho)^{\alpha}\left(1-\rho_{\alpha}\right)} \mathrm{P}(N>x)
$$

as $x \rightarrow \infty$, where $R$ was defined by (2.2).

Remarks. (i) A related result that also allows $Q$ and $N$ to be dependent was derived very recently in [35] using transform methods and Tauberian theorems under the moment conditions $\mathrm{E}[Q]<1$ and $\mathrm{E}[C]=(1-\mathrm{E}[Q]) / \mathrm{E}[N]$.

(ii) Note that this result implies the classical result on the busy period of an M/G/1 queue derived in [13]. Specifically, the total number of customers in a busy period $B$ satisfies the recursion $B \stackrel{\mathrm{D}}{=} 1+\sum_{i=1}^{N(S)} B_{i}$, where the $B_{i}$ s are i.i.d. copies of $B, N(t)$ is a Poisson process of rate $\lambda$, and $S$ is the service distribution; $\left\{B_{i}\right\}, N(t)$, and $S$ are mutually independent, and 
$\rho=\mathrm{E}[N(S)]<1$. Now, the recursion for $B$ is obtained from our theorem by setting $C \equiv 1$ and $Q \equiv 1$, implying that $\mathrm{P}(B>x) \sim \mathrm{P}(N(S)>x) /(1-\rho)^{\alpha+1}$. Next, we can obtain the asymptotics for the length of the busy period $P$ by using the identity $B=N(P)$. This can be easily derived, in spite of the fact that $N(t)$ and $P$ are correlated, since $N(t)$ is highly concentrated around its mean. For recent work on the power law asymptotics of the GI/GI/1 busy period, see [37].

(iii) In view of Lemma 5.1, the theorem shows that the $\operatorname{limits}_{\lim } \rightarrow \infty \lim _{n \rightarrow \infty} \mathrm{P}\left(R^{(n)}>\right.$ $x) / \mathrm{P}(N>x)$ are interchangeable.

Proof of Theorem 5.1. Fix $0<\delta<1$ and $n_{0} \geq 1$. Choose $\rho \vee \rho_{\alpha}<\eta<1$, and use Proposition 5.1 to obtain, for some constant $K_{0}>0$,

$$
\mathrm{P}\left(W_{n}>x\right) \leq K_{0} \eta^{n} \mathrm{P}(N>x)
$$

for all $n \geq 1$ and all $x \geq 1$. Let $H_{\alpha}^{(n)}=(\mathrm{E}[C] \mathrm{E}[Q])^{\alpha}(1-\rho)^{-\alpha} \sum_{k=0}^{n} \rho_{\alpha}^{k}\left(1-\rho^{n-k}\right)^{\alpha}$ and $H_{\alpha}=H_{\alpha}^{(\bar{\infty})}$. Then

$$
\begin{aligned}
\left|\mathrm{P}(R>x)-H_{\alpha} \mathrm{P}(N>x)\right| \leq & \left|\mathrm{P}(R>x)-\mathrm{P}\left(R^{\left(n_{0}\right)}>x\right)\right| \\
& +\left|\mathrm{P}\left(R^{\left(n_{0}\right)}>x\right)-H_{\alpha}^{\left(n_{0}\right)} \mathrm{P}(N>x)\right| \\
& +\left|H_{\alpha}^{\left(n_{0}\right)}-H_{\alpha}\right| \mathrm{P}(N>x) .
\end{aligned}
$$

By Lemma 5.1, there exists a function $\varphi(x) \downarrow 0$ as $x \rightarrow \infty$ such that

$$
\left|\mathrm{P}\left(R^{\left(n_{0}\right)}>x\right)-H_{\alpha}^{\left(n_{0}\right)} \mathrm{P}(N>x)\right| \leq \varphi(x) H_{\alpha} \mathrm{P}(N>x) .
$$

To bound (5.3), let $\beta=\eta^{1 /(2 \alpha+2)}<1$ and note that

$$
\begin{aligned}
\mid \mathrm{P}(R> & x)-\mathrm{P}\left(R^{\left(n_{0}\right)}>x\right) \mid \\
\leq & \mathrm{P}\left(R^{\left(n_{0}\right)}+\left(R-R^{\left(n_{0}\right)}\right)>x, R-R^{\left(n_{0}\right)} \leq \delta x\right)-\mathrm{P}\left(R^{\left(n_{0}\right)}>x\right)+\mathrm{P}\left(R-R^{\left(n_{0}\right)}>\delta x\right) \\
\leq & \mathrm{P}\left(R^{\left(n_{0}\right)}>(1-\delta) x\right)-\mathrm{P}\left(R^{\left(n_{0}\right)}>x\right)+\mathrm{P}\left(\sum_{n=n_{0}+1}^{\infty} W_{n}>\delta x\right) \\
\leq & \mathrm{P}\left(R^{\left(n_{0}\right)}>(1-\delta) x\right)-H_{\alpha}^{\left(n_{0}\right)} \mathrm{P}(N>(1-\delta) x)+H_{\alpha}^{\left(n_{0}\right)} \mathrm{P}(N>x)-\mathrm{P}\left(R^{\left(n_{0}\right)}>x\right) \\
& +H_{\alpha}^{\left(n_{0}\right)} \mathrm{P}(N>(1-\delta) x)-H_{\alpha}^{\left(n_{0}\right)} \mathrm{P}(N>x)+\sum_{n=n_{0}+1}^{\infty} \mathrm{P}\left(W_{n}>\delta x(1-\beta) \beta^{n-n_{0}-1}\right) \\
\leq & \left\{2 \varphi((1-\delta) x) \frac{\mathrm{P}(N>(1-\delta) x)}{\mathrm{P}(N>x)}+\left(\frac{\mathrm{P}(N>(1-\delta) x)}{\mathrm{P}(N>x)}-1\right)\right\} H_{\alpha} \mathrm{P}(N>x) \\
& +\sum_{n=n_{0}+1}^{\infty} K_{0} \eta^{n} \mathrm{P}\left(N>\delta x(1-\beta) \beta^{n-n_{0}-1}\right),
\end{aligned}
$$

where in the last inequality we applied the uniform bound from Proposition 5.1. The expression in curly brackets is bounded by

$$
2 \varphi((1-\delta) x)(1-\delta)^{-\alpha} \frac{L((1-\delta) x)}{L(x)}+\left((1-\delta)^{-\alpha} \frac{L((1-\delta) x)}{L(x)}-1\right) \rightarrow(1-\delta)^{-\alpha}-1
$$

as $x \rightarrow \infty$. By Potter's theorem (see Theorem 1.5.6(ii) of [8, p. 25]), there exists a constant 
$A=A(1)>1$ such that

$$
\begin{aligned}
& \sum_{n=n_{0}+1}^{\infty} K_{0} \eta^{n} \mathrm{P}\left(N>\delta x(1-\beta) \beta^{n-n_{0}-1}\right) \\
& \quad \leq K_{0} A \sum_{n=n_{0}+1}^{\infty} \eta^{n}\left(\delta(1-\beta) \beta^{n-n_{0}-1}\right)^{-\alpha-1} \mathrm{P}(N>x) \\
& \quad=K_{0} A(\delta(1-\beta))^{-\alpha-1}\left(1-\eta^{1 / 2}\right)^{-1} \eta^{n_{0}+1} \mathrm{P}(N>x) \\
& \quad \leq K \delta^{-\alpha-1} \eta^{n_{0}} \mathrm{P}(N>x)
\end{aligned}
$$

Next, for (5.5), simply note that

$$
\begin{aligned}
\frac{1}{H_{\alpha}} \mid & H_{\alpha}^{\left(n_{0}\right)}-H_{\alpha} \mid \\
& =\left(1-\rho_{\alpha}\right)\left(\sum_{k=0}^{\infty} \rho_{\alpha}^{k}-\sum_{k=0}^{n_{0}} \rho_{\alpha}^{k}\left(1-\rho^{n_{0}-k}\right)^{\alpha}\right) \\
& =\left(1-\rho_{\alpha}\right) \sum_{k=0}^{n_{0}} \rho_{\alpha}^{k}\left(1-\left(1-\rho^{n_{0}-k}\right)^{\alpha}\right)+\left(1-\rho_{\alpha}\right) \sum_{k=n_{0}+1}^{\infty} \rho_{\alpha}^{k} \\
& \leq\left(1-\rho_{\alpha}\right) \sum_{k=0}^{n_{0}} \rho_{\alpha}^{k} \alpha \rho^{n_{0}-k}+\rho_{\alpha}^{n_{0}+1} \\
& \leq\left[\alpha\left(1-\rho_{\alpha}\right)\left(n_{0}+1\right)+\rho_{\alpha}\right]\left(\rho_{\alpha} \vee \rho\right)^{n_{0}} \\
& \leq K \eta^{n_{0}} .
\end{aligned}
$$

Finally, by replacing the preceding estimates in (5.3)-(5.5), we obtain

$$
\lim _{x \rightarrow \infty}\left|\frac{\mathrm{P}(R>x)}{H_{\alpha} \mathrm{P}(N>x)}-1\right| \leq(1-\delta)^{-\alpha}-1+K \delta^{-\alpha-1} \eta^{n_{0}} .
$$

Since the right-hand side can be made arbitrarily small by first letting $n_{0} \rightarrow \infty$ and then $\delta \downarrow 0$, the result of the theorem follows.

\subsection{Engineering implications}

Recall that, for Google's PageRank algorithm, the weights are given by $C_{i}=c / D_{i}<1$, where $0<c<1$ is a constant related to the damping factor and the number of nodes in the Web graph, and $D_{i}$ corresponds to the out-degree of a page. We point out that dividing the ranks of neighboring pages by their out-degree has the purpose of decreasing the contribution of pages with highly inflated referencing. However, Theorem 5.1 reveals that the page rank is essentially insensitive to the parameters of the out-degree distribution, which means that PageRank basically reflects the popularity vote given by the number of references $N$. This same observation was previously made in [35].

Furthermore, Theorem 4.1 clearly shows that the choice of weights $C_{i}$ in the ranking algorithm can determine the distribution of $R$ as well. Note that, for the PageRank algorithm, the weights $C_{i}=c / D_{i}<1$ can never dominate the asymptotic behavior of $R$ when $N$ is a power law. Therefore, Theorem 4.1 suggests a potential development of new ranking algorithms where the ranks will be much more sensitive to the weights. 


\section{The case when $Q$ dominates}

This section of the paper treats the case when the heavy-tailed behavior of $R$ arises from the $\left\{Q_{i}\right\}$, known in the autoregressive processes literature as innovations. The results presented here are very similar to those in Section 5, and so are their proofs. We will therefore only present the statements of the results and skip most of the proofs. We start with the equivalents of Lemmas 5.1 and 5.2 in this context; similarly as in Section 5, the proofs are standard and can be found in the preprint version of this paper [20].

Lemma 6.1. Suppose that $\mathrm{P}(Q>x)=x^{-\alpha} L(x)$ with $L(\cdot)$ slowly varying, $\alpha>1$, and $\mathrm{E}\left[N^{\alpha+\epsilon}\right]<\infty, \mathrm{E}\left[C^{\alpha+\epsilon}\right]<\infty$ for some $\epsilon>0$; let $\rho_{\alpha}=\mathrm{E}[N] \mathrm{E}\left[C^{\alpha}\right]$. Then, for any fixed $n \in\{1,2,3, \ldots\}$,

$$
\mathrm{P}\left(R^{(n)}>x\right) \sim \sum_{k=0}^{n} \rho_{\alpha}^{k} \mathrm{P}(Q>x)
$$

as $x \rightarrow \infty$, where $R^{(n)}$ was defined in Section 2.1 .

As for the case when $N$ dominates the asymptotic behavior of $R$, we can here expect that

$$
\mathrm{P}(R>x) \sim\left(1-\rho_{\alpha}\right)^{-1} \mathrm{P}(Q>x),
$$

and the technical difficulty is justifying the exchange of limits. The same techniques used in Section 5 can be used in this case as well. Therefore, we give a sketch of the arguments in Section 7.4 but omit the proof. The following is the equivalent of Lemma 5.2.

Lemma 6.2. Suppose that $\mathrm{P}(Q>x)=x^{-\alpha} L(x)$ with $L(\cdot)$ slowly varying, $\alpha>1$, and $\mathrm{E}\left[N^{\alpha+\epsilon}\right]<\infty, \mathrm{E}\left[C^{\alpha+\epsilon}\right]<\infty$ for some $\epsilon>0$; let $\rho_{\alpha}=\mathrm{E}[N] \mathrm{E}\left[C^{\alpha}\right]$. Then, for any fixed $n \in\{1,2,3, \ldots\}$,

$$
\mathrm{P}\left(W_{n}>x\right) \sim \rho_{\alpha}^{n} \mathrm{P}(Q>x)
$$

as $x \rightarrow \infty$, where $W_{n}$ was defined in Section 2.1.

The corresponding version of Proposition 5.1 is given below.

Proposition 6.1. Suppose that $\mathrm{P}(Q>x)=x^{-\alpha} L(x)$ with $L(\cdot)$ slowly varying, $\alpha>1$, and $\mathrm{E}\left[C^{\alpha+v}\right]<\infty, \mathrm{E}\left[N^{\alpha+v}\right]<\infty$ for some $v>0$, and let $\mathrm{E}[N] \max \left\{\mathrm{E}\left[C^{\alpha}\right], \mathrm{E}[C]\right\}<\eta<1$. Then, there exists a constant $K=K(\eta, v)>0$ such that, for all $n \geq 1$ and all $x \geq 1$,

$$
\mathrm{P}\left(W_{n}>x\right) \leq K \eta^{n} \mathrm{P}(Q>x) .
$$

A sketch of the proof can be found in Section 7.4.

Finally, we present the main theorem of this section. The proof again greatly resembles that of Theorem 5.1 and is therefore omitted.

Theorem 6.1. Suppose that $\mathrm{P}(Q>x)=x^{-\alpha} L(x)$ with $L(\cdot)$ slowly varying and $\alpha>1$. Let $\rho=\mathrm{E}[N] \mathrm{E}[C]$ and $\rho_{\alpha}=\mathrm{E}[N] \mathrm{E}\left[C^{\alpha}\right]$. Assume that $\rho \vee \rho_{\alpha}<1$, and that $\mathrm{E}\left[C^{\alpha+\epsilon}\right]<\infty$ and $\mathrm{E}\left[N^{\alpha+\epsilon}\right]<\infty$ for some $\epsilon>0$. Then

$$
\mathrm{P}(R>x) \sim\left(1-\rho_{\alpha}\right)^{-1} \mathrm{P}(Q>x)
$$

as $x \rightarrow \infty$, where $R$ was defined by (2.2). 
Compare this result with Lemma A.3 of [29], where the autoregressive process of order 1 with regularly varying innovations is shown to be tail equivalent to $Q$. In particular, if we set $N \equiv 1$ in Theorem 6.1 and let $A_{k}=\prod_{i=1}^{k-1} C_{i}$, our result reduces to

$$
\mathrm{P}\left(\sum_{k=0}^{\infty} A_{k} Q_{k}>x\right) \sim \sum_{k=0}^{\infty} \mathrm{E}\left[A_{k}^{\alpha}\right] \mathrm{P}(Q>x),
$$

which is in line with the commonly accepted intuition about heavy-tailed large deviations where large sums are due to one large summand $Q_{k}$.

\section{Proofs}

This section contains the proofs to most of the technical results presented in the paper, together with some auxiliary lemmas that are needed along the way. The section is divided into four subsections, each corresponding to the content of Sections 3, 4, 5, and 6, respectively.

\subsection{Moments of $\boldsymbol{W}_{\boldsymbol{n}}$}

Here we give the proof of the moment bound for the $\beta$-moment, $\beta>1$, of the sum of the weights, $W_{n}$ of the $n$th generation. As an intermediate step, we present a lemma for the integer moments of $W_{n}$, but first we give the proof of Lemma 4.2.

Proof of Lemma 4.2. Let $p=\lceil\beta\rceil \in\{2,3, \ldots\}$ and $\gamma=\beta / p \in(0,1]$. Define $A_{p}(k)=$ $\left\{\left(j_{1}, \ldots, j_{k}\right) \in \mathbb{Z}^{k}: j_{1}+\cdots+j_{k}=p, 0 \leq j_{i}<p\right\}$. Then

$$
\begin{aligned}
\left(\sum_{i=1}^{k} y_{i}\right)^{\beta} & =\left(\sum_{i=1}^{k} y_{i}\right)^{p \gamma} \\
& =\left(\sum_{i=1}^{k} y_{i}^{p}+\sum_{\left(j_{1}, \ldots, j_{k}\right) \in A_{p}(k)}\left(\begin{array}{c}
p \\
j_{1}, \ldots, j_{k}
\end{array}\right) y_{1}^{j_{1}} \cdots y_{k}^{j_{k}}\right)^{\gamma} \\
& \leq \sum_{i=1}^{k} y_{i}^{p \gamma}+\left(\sum_{\left(j_{1}, \ldots, j_{k}\right) \in A_{p}(k)}\left(\begin{array}{c}
p \\
j_{1}, \ldots, j_{k}
\end{array}\right) y_{1}^{j_{1}} \cdots y_{k}^{j_{k}}\right)^{\gamma},
\end{aligned}
$$

where, for the last step, we used the well-known inequality $\left(\sum_{i=1}^{k} x_{i}\right)^{\gamma} \leq \sum_{i=1}^{k} x_{i}^{\gamma}$ for $0<$ $\gamma \leq 1$ and $x_{i} \geq 0$ (see the proof of Lemma 3.1). We now use Jensen's inequality to obtain

$$
\begin{aligned}
\mathrm{E}\left[\left(\sum_{i=1}^{k} Y_{i}\right)^{\beta}-\sum_{i=1}^{k} Y_{i}^{\beta}\right] & \leq \mathrm{E}\left[\left(\sum_{\left(j_{1}, \ldots, j_{k}\right) \in A_{p}(k)}\left(\begin{array}{c}
p \\
j_{1}, \ldots, j_{k}
\end{array}\right) Y_{1}^{j_{1}} \cdots Y_{k}^{j_{k}}\right)^{\gamma}\right] \\
& \leq\left(\mathrm{E}\left[\sum_{\left(j_{1}, \ldots, j_{k}\right) \in A_{p}(k)}\left(\begin{array}{c}
p \\
j_{1}, \ldots, j_{k}
\end{array}\right) Y_{1}^{j_{1}} \cdots Y_{k}^{j_{k}}\right]\right)^{\gamma} \\
& =\left(\sum_{\left(j_{1}, \ldots, j_{k}\right) \in A_{p}(k)}\left(\begin{array}{c}
p \\
j_{1}, \ldots, j_{k}
\end{array}\right) \mathrm{E}\left[Y_{1}^{j_{1}} \cdots Y_{k}^{j_{k}}\right]\right)^{\gamma} .
\end{aligned}
$$

Since the $\left\{Y_{i}\right\}$ are i.i.d., we have

$$
\mathrm{E}\left[Y_{1}^{j_{1}} \cdots Y_{k}^{j_{k}}\right]=\|Y\|_{j_{1}}^{j_{1}} \cdots\|Y\|_{j_{k}}^{j_{k}}
$$

where $\|Y\|_{\kappa}=\mathrm{E}\left[|Y|^{\kappa}\right]^{1 / \kappa}$ for $\kappa \geq 1$ and $\|Y\|_{0}:=1$. Since $\|Y\|_{\kappa}$ is increasing for $\kappa \geq 1$, it 
follows that $\|Y\|_{j_{i}}^{j_{i}} \leq\|Y\|_{p-1}^{j_{1}}$. Furthermore, it follows that

$$
\|Y\|_{j_{1}}^{j_{1}} \cdots\|Y\|_{j_{k}}^{j_{k}} \leq\|Y\|_{p-1}^{p},
$$

which in turn implies that

$$
\begin{aligned}
\mathrm{E}\left[\left(\sum_{i=1}^{k} Y_{i}\right)^{\beta}-\sum_{i=1}^{k} Y_{i}^{\beta}\right] & \leq\left(\sum_{\left(j_{1}, \ldots, j_{k}\right) \in A_{p}(k)}\left(\begin{array}{c}
p \\
j_{1}, \ldots, j_{k}
\end{array}\right)\|Y\|_{p-1}^{p}\right)^{\gamma} \\
& =\|Y\|_{p-1}^{\beta}\left(k^{p}-k\right)^{\gamma} \\
& \leq\|Y\|_{p-1}^{\beta} k^{\beta} .
\end{aligned}
$$

Lemma 7.1. Suppose that $\mathrm{E}\left[Q^{p}\right]<\infty, \mathrm{E}\left[N^{p}\right]<\infty$, and $\mathrm{E}[N] \max \left\{\mathrm{E}\left[C^{p}\right], \mathrm{E}[C]\right\}<1$ for some $p \in\{2,3, \ldots\}$. Then, there exists a constant $K_{p}>0$ such that

$$
\mathrm{E}\left[W_{n}^{p}\right] \leq K_{p}\left(\mathrm{E}[N] \max \left\{\mathrm{E}[C], \mathrm{E}\left[C^{p}\right]\right\}\right)^{n}
$$

for all $n \geq 0$.

Proof. Let $Y=C W_{n-1}$, where $C$ is independent of $W_{n-1}$, and let $\left\{Y_{i}\right\}$ be independent copies of $Y$. We will give an induction proof in $p$. For $p=2$, we have

$$
\begin{aligned}
\mathrm{E}\left[W_{n}^{2}\right] & =\mathrm{E}\left[\left(\sum_{i=1}^{N} Y_{i}\right)^{2}\right] \\
& =\mathrm{E}[N] \mathrm{E}\left[Y^{2}\right]+\mathrm{E}[N(N-1)](\mathrm{E}[Y])^{2} \\
& =\mathrm{E}[N] \mathrm{E}\left[C^{2}\right] \mathrm{E}\left[W_{n-1}^{2}\right]+\mathrm{E}[N(N-1)]\left(\mathrm{E}[C] \mathrm{E}\left[W_{n-1}\right]\right)^{2} .
\end{aligned}
$$

Using the preceding recursion, letting $\rho=\mathrm{E}[N] \mathrm{E}[C]$ and $\rho_{2}=\mathrm{E}[N] \mathrm{E}\left[C^{2}\right]$, and noting that

$$
\mathrm{E}\left[W_{n-1}\right]=\rho^{n-1} \mathrm{E}[Q],
$$

we obtain

$$
\mathrm{E}\left[W_{n}^{2}\right]=\rho_{2} \mathrm{E}\left[W_{n-1}^{2}\right]+K \rho^{2(n-1)},
$$

where $K=\mathrm{E}[N(N-1)](\mathrm{E}[C] \mathrm{E}[Q])^{2}$. Now, iterating (7.1) gives the result for $p=2$.

For any $p \in\{2,3, \ldots\}$, the result can be obtained inductively using Lemma 4.2 and standard algebra, and, thus, we present the details in the preprint version of this paper [20].

The proof for the general $\beta$-moment, $\beta>1$, is given below.

Proof of Lemma 3.2. Set $p=\lceil\beta\rceil \geq \beta>1$. Since the result when $p=\beta$ follows from Lemma 7.1, we assume that $p>\beta$. Let $Y=C W_{n-1}$, where $C$ is independent of $W_{n-1}$, and let $\left\{Y_{i}\right\}$ be independent copies of $Y$. Also, recall that $\rho=\mathrm{E}[N] \mathrm{E}[C]$ and $\rho_{\beta}=\mathrm{E}[N] \mathrm{E}\left[C^{\beta}\right]$. Then, by Lemma 4.2,

$$
\begin{aligned}
\mathrm{E}\left[W_{n}^{\beta}\right] & =\mathrm{E}\left[\left(\sum_{i=1}^{N} Y_{i}\right)^{\beta}\right] \\
& =\sum_{k=1}^{\infty} \mathrm{E}\left[\left(\sum_{i=1}^{k} Y_{i}\right)^{\beta}\right] \mathrm{P}(N=k) \\
& =\sum_{k=1}^{\infty}\left(\mathrm{E}\left[\left(\sum_{i=1}^{k} Y_{i}\right)^{\beta}-\sum_{i=1}^{k} Y_{i}^{\beta}\right]+\mathrm{E}\left[\sum_{i=1}^{k} Y_{i}^{\beta}\right]\right) \mathrm{P}(N=k)
\end{aligned}
$$




$$
\begin{aligned}
& \leq \sum_{k=1}^{\infty}\left(k^{\beta} \mathrm{E}\left[Y^{p-1}\right]^{\beta /(p-1)}+k \mathrm{E}\left[Y^{\beta}\right]\right) \mathrm{P}(N=k) \\
& =\mathrm{E}\left[N^{\beta}\right]\left(\mathrm{E}\left[C^{p-1}\right]\right)^{\beta /(p-1)}\left(\mathrm{E}\left[W_{n-1}^{p-1}\right]\right)^{\beta /(p-1)}+\rho_{\beta} \mathrm{E}\left[W_{n-1}^{\beta}\right] .
\end{aligned}
$$

Then, by Lemma 7.1,

$$
\begin{aligned}
\mathrm{E}\left[W_{n}^{\beta}\right] & \leq \rho_{\beta} \mathrm{E}\left[W_{n-1}^{\beta}\right]+\mathrm{E}\left[N^{\beta}\right]\left(\mathrm{E}\left[C^{p-1}\right]\right)^{\beta /(p-1)}\left(K_{p-1}\left(\rho_{p-1} \vee \rho\right)^{n-1}\right)^{\beta /(p-1)} \\
& =\rho_{\beta} \mathrm{E}\left[W_{n-1}^{\beta}\right]+K\left(\rho_{p-1} \vee \rho\right)^{(n-1) \gamma},
\end{aligned}
$$

where $\gamma=\beta /(p-1)>1$. Finally, iterating the preceding bound $n-1$ times gives

$$
\begin{aligned}
\mathrm{E}\left[W_{n}^{\beta}\right] & \leq \rho_{\beta}^{n} \mathrm{E}\left[W_{0}^{\beta}\right]+K \sum_{i=0}^{n-1} \rho_{\beta}^{i}\left(\rho \vee \rho_{p-1}\right)^{\gamma(n-1-i)} \\
& \leq \mathrm{E}\left[W_{0}^{\beta}\right]\left(\rho \vee \rho_{\beta}\right)^{n}+K \sum_{i=0}^{n-1}\left(\rho \vee \rho_{\beta}\right)^{\gamma(n-1-i)+i} \\
& =\mathrm{E}\left[Q^{\beta}\right]\left(\rho \vee \rho_{\beta}\right)^{n}+K\left(\rho \vee \rho_{\beta}\right)^{n-1} \sum_{i=0}^{n-1}\left(\rho \vee \rho_{\beta}\right)^{(\gamma-1) i} \\
& \leq K_{\beta}\left(\rho \vee \rho_{\beta}\right)^{n} .
\end{aligned}
$$

This completes the proof.

\subsection{The case when the $C$ s dominate: implicit renewal theory}

In this section we state a lemma that is used in the proof of Theorem 4.1, and we give the proofs to Theorem 4.1 and Lemma 4.3.

Lemma 7.2. Let $\alpha, \beta>0$ and $H \geq 0$. Suppose that $\int_{0}^{t} v^{\alpha+\beta-1} \mathrm{P}(R>v) \mathrm{d} v \sim H t^{\beta} / \beta$ as $t \rightarrow \infty$. Then

$$
\mathrm{P}(R>t) \sim H t^{-\alpha}, \quad t \rightarrow \infty .
$$

Proof. This lemma is a special case of the monotone density theorem; see Theorem 1.7.5 (also Exercise 1.11.14) of [8]. However, for completeness, we give a direct proof here, similar to that of Lemma 9.3 of [16]. By assumption, for any $b>1, \epsilon \in(0,1)$, and sufficiently large $t$,

$$
\begin{aligned}
\mathrm{P}(R>t) t^{\alpha+\beta} \frac{b^{\alpha+\beta}-1}{\alpha+\beta} & \geq \int_{t}^{b t} v^{\alpha+\beta-1} \mathrm{P}(R>v) \mathrm{d} v \\
& \geq \frac{H-\epsilon}{\beta}(b t)^{\beta}-\frac{H+\epsilon}{\beta} t^{\beta} \\
& \geq \frac{t^{\beta}}{\beta}\left(H\left(b^{\beta}-1\right)-\epsilon\left(1+b^{\beta}\right)\right) .
\end{aligned}
$$

Since $\epsilon$ was arbitrary, we can take the limit as $\epsilon \rightarrow 0$ and obtain

$$
\liminf _{t \rightarrow \infty} \mathrm{P}(R>t) t^{\alpha} \geq \frac{H(\alpha+\beta)\left(b^{\beta}-1\right)}{\beta\left(b^{\alpha+\beta}-1\right)} \rightarrow H, \quad b \downarrow 1 .
$$

Similarly, we can prove that $\lim \sup _{t \rightarrow \infty} \mathrm{P}(R>t) t^{\alpha} \leq H$ starting from $\int_{b t}^{t} v^{\alpha+\beta-1} \mathrm{P}(R>v) \mathrm{d} v$ with $0<b<1$. 
Proof of Theorem 4.1. For any $k \in \mathbb{N}$, define $\Pi_{k}=\prod_{i=1}^{k} C_{i}$ and $V_{k}=\sum_{i=1}^{k} \log C_{i}$, with $\Pi_{0}=1$ and $V_{0}=0$, where the $C_{i} \mathrm{~s}$ are independent copies of $C$. Then, for any $t \in \mathbb{R}$,

$$
\begin{aligned}
\mathrm{P}\left(R>\mathrm{e}^{t}\right)= & \sum_{k=1}^{n}\left(m^{k-1} \mathrm{P}\left(\Pi_{k-1} R>\mathrm{e}^{t}\right)-m^{k} \mathrm{P}\left(\Pi_{k} R>\mathrm{e}^{t}\right)\right)+m^{n} \mathrm{P}\left(\Pi_{n} R>\mathrm{e}^{t}\right) \\
= & \sum_{k=1}^{n}\left(m^{k-1} \mathrm{P}\left(\mathrm{e}^{V_{k-1}} R>\mathrm{e}^{t}\right)-m^{k} \mathrm{P}\left(\mathrm{e}^{V_{k-1}} C_{k} R>\mathrm{e}^{t}\right)\right)+m^{n} \mathrm{P}\left(\mathrm{e}^{V_{n}} R>\mathrm{e}^{t}\right) \\
= & \sum_{k=0}^{n-1} m^{k} \int_{-\infty}^{\infty}\left(\mathrm{P}\left(R>\mathrm{e}^{t-v}\right)-m \mathrm{P}\left(C R>\mathrm{e}^{t-v}\right)\right) \mathrm{P}\left(V_{k} \in \mathrm{d} v\right) \\
& +m^{n} \mathrm{P}\left(\mathrm{e}^{V_{n}} R>\mathrm{e}^{t}\right) .
\end{aligned}
$$

Next, define

$$
\begin{gathered}
v_{n}(\mathrm{~d} t)=\mathrm{e}^{\alpha t} \sum_{k=0}^{n} m^{k} \mathrm{P}\left(V_{k} \in \mathrm{d} t\right), \quad g(t)=\mathrm{e}^{\alpha t}\left(\mathrm{P}\left(R>\mathrm{e}^{t}\right)-m \mathrm{P}\left(C R>\mathrm{e}^{t}\right)\right), \\
r(t)=\mathrm{e}^{\alpha t} \mathrm{P}\left(R>\mathrm{e}^{t}\right), \quad \text { and } \quad \delta_{n}(t)=m^{n} \mathrm{P}\left(\mathrm{e}^{V_{n}} R>\mathrm{e}^{t}\right) .
\end{gathered}
$$

Then, for any $t \in \mathbb{R}$ and $n \in \mathbb{N}$,

$$
r(t)=\left(g * v_{n-1}\right)(t)+\delta_{n}(t) .
$$

Next, for any $\beta>0$, define the smoothing operator

$$
\breve{f}(t)=\int_{-\infty}^{t} \mathrm{e}^{-\beta(t-u)} f(u) \mathrm{d} u
$$

and note that

$$
\begin{aligned}
\breve{r}(t) & =\int_{-\infty}^{t} \mathrm{e}^{-\beta(t-u)}\left(g * v_{n-1}\right)(u) \mathrm{d} u+\breve{\delta}_{n}(t) \\
& =\int_{-\infty}^{t} \mathrm{e}^{-\beta(t-u)} \int_{-\infty}^{\infty} g(u-v) v_{n-1}(\mathrm{~d} v) \mathrm{d} u+\breve{\delta}_{n}(t) \\
& =\int_{-\infty}^{\infty} \int_{-\infty}^{t} \mathrm{e}^{-\beta(t-u)} g(u-v) \mathrm{d} u v_{n-1}(\mathrm{~d} v)+\breve{\delta}_{n}(t) \\
& =\int_{-\infty}^{\infty} \breve{g}(t-v) v_{n-1}(\mathrm{~d} v)+\breve{\delta}_{n}(t) \\
& =\left(\breve{g} * v_{n-1}\right)(t)+\breve{\delta}_{n}(t) .
\end{aligned}
$$

Next, we will show that we can pass $n \rightarrow \infty$ in the preceding identity. To this end, let $\eta(\mathrm{d} u)=\mathrm{e}^{\alpha u} m \mathrm{P}(\log C \in \mathrm{d} u)$, and note that this measure places no mass at $-\infty$. Also, by assumption, $\eta(\cdot)$ is a nonarithmetic measure on $\mathbb{R}$. Moreover,

$$
\int_{-\infty}^{\infty} \eta(\mathrm{d} u)=m \mathrm{E}\left[\mathrm{e}^{\alpha \log C}\right]=m \mathrm{E}\left[C^{\alpha}\right]=1
$$

and

$$
\int_{-\infty}^{\infty} u \eta(\mathrm{d} u)=m \mathrm{E}\left[\mathrm{e}^{\alpha \log C} \log C\right]=m \mathrm{E}\left[C^{\alpha} \log C\right]=m \mu
$$


imply that $\eta(\cdot)$ is a probability measure with mean $0<m \mu<\infty$. Furthermore,

$$
v(\mathrm{~d} t)=\sum_{k=0}^{\infty} m^{k} \mathrm{e}^{\alpha t} \mathrm{P}\left(V_{k} \in \mathrm{d} t\right)
$$

is its renewal measure since $v(\mathrm{~d} t)=\sum_{n=0}^{\infty} \eta^{* n}(\mathrm{~d} t)$. Since $m \mu>0$, then $(|f| * v)(t)<\infty$ for all $t$ whenever $f$ is directly Riemann integrable. From (4.2) we know that $g \in L^{1}$, so, by Lemma 9.2 of [16], $\breve{g}$ is directly Riemann integrable, resulting in $(|\breve{g}| * v)(t)<\infty$ for all $t$. Thus, $(|\breve{g}| * v)(t)=\mathrm{E}\left[\sum_{k=0}^{\infty} m^{k} \mathrm{e}^{\alpha V_{k}}\left|\breve{g}\left(t-V_{k}\right)\right|\right]<\infty$. By Fubini's theorem, E[ $\sum_{k=0}^{\infty} m^{k} \mathrm{e}^{\alpha V_{k}} \breve{g}(t-$ $\left.V_{k}\right)$ ] exists and

$$
(\breve{g} * v)(t)=\mathrm{E}\left[\sum_{k=0}^{\infty} m^{k} \mathrm{e}^{\alpha V_{k}} \breve{g}\left(t-V_{k}\right)\right]=\sum_{k=0}^{\infty} \mathrm{E}\left[m^{k} \mathrm{e}^{\alpha V_{k}} \breve{g}\left(t-V_{k}\right)\right]=\lim _{n \rightarrow \infty}\left(\breve{g} * v_{n}\right)(t) .
$$

Now, by assumption we can choose $\beta$ in the definition of the smoothing operator such that $0<\beta<\alpha$ and $m \mathrm{E}\left[C^{\beta}\right]<1$. We show below that, for such $\beta$, we have $\breve{\delta}_{n}(t) \rightarrow 0$ as $n \rightarrow \infty$ for all fixed $t$, since

$$
\begin{aligned}
\breve{\delta}_{n}(t) & =\int_{-\infty}^{t} \mathrm{e}^{-\beta(t-u)} m^{n} \mathrm{P}\left(\mathrm{e}^{\beta V_{n}} R^{\beta}>\mathrm{e}^{\beta u}\right) \mathrm{d} u \\
& =\frac{\mathrm{e}^{-\beta t} m^{n}}{\beta} \int_{0}^{\mathrm{e}^{\beta t}} \mathrm{P}\left(\mathrm{e}^{\beta V_{n}} R^{\beta}>v\right) \mathrm{d} v \\
& \leq \frac{\mathrm{e}^{-\beta t}}{\beta} \mathrm{E}\left[R^{\beta}\right]\left(m \mathrm{E}\left[C^{\beta}\right]\right)^{n} \\
& \rightarrow 0 \quad \text { as } n \rightarrow \infty .
\end{aligned}
$$

Hence, the preceding arguments allow us to pass $n \rightarrow \infty$ in (7.2), and obtain

$$
\breve{r}(t)=(\breve{g} * v)(t) .
$$

Now, by the key renewal theorem for two-sided random walks in [5],

$$
\mathrm{e}^{-\beta t} \int_{0}^{\mathrm{e}^{t}} v^{\alpha+\beta-1} \mathrm{P}(R>v) \mathrm{d} v=\breve{r}(t) \rightarrow \frac{1}{m \mu} \int_{-\infty}^{\infty} \breve{g}(u) \mathrm{d} u=: \frac{H}{\beta}, \quad t \rightarrow \infty .
$$

Clearly, $H \geq 0$ since the left-hand side of the preceding equation is positive, and, thus, by Lemma 7.2,

$$
\mathrm{P}(R>t) \sim H t^{-\alpha}, \quad t \rightarrow \infty .
$$

Finally,

$$
\begin{aligned}
H & =\frac{\beta}{m \mu} \int_{-\infty}^{\infty} \int_{-\infty}^{u} \mathrm{e}^{-\beta(u-t)} g(t) \mathrm{d} t \mathrm{~d} u \\
& =\frac{1}{m \mu} \int_{-\infty}^{\infty} g(t) \mathrm{d} t \\
& =\frac{1}{m \mu} \int_{0}^{\infty} v^{\alpha-1}(\mathrm{P}(R>v)-m \mathrm{P}(C R>v)) \mathrm{d} v
\end{aligned}
$$

We end this section with the proof of Lemma 4.3. 
Proof of Lemma 4.3. That the integral is positive follows from the union bound. That

$$
\begin{gathered}
\int_{0}^{\infty}\left(\mathrm{E}[N] \mathrm{P}(C R>t)-\mathrm{P}\left(\max _{1 \leq i \leq N} C_{i} R_{i}>t\right)\right) t^{\alpha-1} \mathrm{~d} t \\
=\frac{1}{\alpha} \mathrm{E}\left[\sum_{i=1}^{N}\left(C_{i} R_{i}\right)^{\alpha}-\left(\max _{1 \leq i \leq N} C_{i} R_{i}\right)^{\alpha}\right]
\end{gathered}
$$

follows from similar arguments to those used to derive the alternative expression for $H$ in the proof of Theorem 4.2 found in the preprint version of this paper [20]. The rest of the proof shows that the integral is finite.

Clearly,

$$
\int_{0}^{1}\left(\mathrm{E}[N] \mathrm{P}(C R>t)-\mathrm{P}\left(\max _{1 \leq i \leq N} C_{i} R_{i}>t\right)\right) t^{\alpha-1} \mathrm{~d} t \leq \mathrm{E}[N] \int_{0}^{1} t^{\alpha-1} \mathrm{~d} t<\infty .
$$

Hence, it remains to prove that the remaining part of the integral $\left(\int_{1}^{\infty} \cdots \mathrm{d} t\right)$ is finite. To do this, we start by letting $Y=C R$ and $F(y)=\mathrm{P}(Y \leq y)$. Then

$$
\begin{aligned}
\mathrm{E}[N] \mathrm{P}(C R>t)-\mathrm{P}\left(\max _{1 \leq i \leq N} C_{i} R_{i}>t\right) & =\sum_{k=1}^{\infty}\left(F(t)^{k}-1+k \bar{F}(t)\right) \mathrm{P}(N=k) \\
& =\mathrm{E}\left[(1-\bar{F}(t))^{N}-1+N \bar{F}(t)\right] .
\end{aligned}
$$

Use the inequality $1-x \leq \mathrm{e}^{-x}$ for $x \geq 0$ to obtain

$$
\mathrm{E}\left[(1-\bar{F}(t))^{N}-1+N \bar{F}(t)\right] \leq \mathrm{E}\left[\mathrm{e}^{-\bar{F}(t) N}-1+N \bar{F}(t)\right] .
$$

Choose $0<\delta<\alpha \epsilon /(1+\epsilon)$ (recall that $0<\epsilon<1$ ), and let $\beta=\alpha-\delta$. By Markov's inequality and Lemma 3.3,

$$
\bar{F}(t) \leq t^{-\beta} \mathrm{E}\left[Y^{\beta}\right]=t^{-\beta} \mathrm{E}\left[R^{\beta}\right] \mathrm{E}\left[C^{\beta}\right]=: c t^{-\beta}<\infty
$$

for any $t>0$. Note that the function $h(x)=\mathrm{e}^{-x}-1+x$ is increasing on $[0, \infty)$, so

$$
h(N \bar{F}(t)) \leq h\left(c N t^{-\beta}\right) .
$$

Thus, by Fubini's theorem (the integrand is nonnegative),

$$
\begin{gathered}
\int_{1}^{\infty}\left(\mathrm{E}[N] \mathrm{P}(C R>t)-\mathrm{P}\left(\max _{1 \leq i \leq N} C_{i} R_{i}>t\right)\right) t^{\alpha-1} \mathrm{~d} t \\
\leq \mathrm{E}\left[\int_{1}^{\infty}\left(\mathrm{e}^{-c N t^{-\beta}}-1+c N t^{-\beta}\right) t^{\alpha-1} \mathrm{~d} t\right] .
\end{gathered}
$$

Using the change of variable $u=c N t^{-\beta}$ gives

$$
\begin{aligned}
\int_{1}^{\infty}\left(\mathrm{e}^{-c N t^{-\beta}}-1+c N t^{-\beta}\right) t^{\alpha-1} \mathrm{~d} t & =\frac{(c N)^{\alpha / \beta}}{\beta} \int_{0}^{c N}\left(\mathrm{e}^{-u}-1+u\right) u^{-\alpha / \beta-1} \mathrm{~d} u \\
& \leq \frac{(c N)^{\alpha / \beta}}{\beta} \int_{0}^{\infty}\left(\mathrm{e}^{-u}-1+u\right) u^{-\alpha / \beta-1} \mathrm{~d} u
\end{aligned}
$$


Our choice of $\beta=\alpha-\delta$ guarantees that $1<\alpha / \beta<1+\epsilon$, so $\mathrm{E}\left[(c N)^{\alpha / \beta}\right]<\infty$. It only remains to show that the last (nonrandom) integral is finite. To see this, note that $\mathrm{e}^{-x}-1+x \leq x^{2} / 2$ and $\mathrm{e}^{-x}-1 \leq 0$ for any $x \geq 0$, so

$$
\begin{aligned}
\int_{0}^{\infty}\left(\mathrm{e}^{-u}-1+u\right) u^{-\alpha / \beta-1} \mathrm{~d} u & \leq \frac{1}{2} \int_{0}^{1} u^{1-\alpha / \beta} \mathrm{d} u+\int_{1}^{\infty} u^{-\alpha / \beta} \mathrm{d} u \\
& =\frac{1}{2(2-\alpha / \beta)}+\frac{1}{\alpha / \beta-1} \\
& <\infty
\end{aligned}
$$

This completes the proof.

\subsection{The case when $N$ dominates}

This section contains the proof of Proposition 5.1. We also present in Lemma 7.3 a result for sums of i.i.d. truncated random variables that may be of independent interest in the context of heavy-tailed asymptotics, since it provides bounds that do not depend on the distribution of the summands. Most of the work involved in the proof of Proposition 5.1 goes into obtaining a bound for one iteration of the recursion satisfied by $W_{n}$, and, for the convenience of the reader, it is presented separately in Lemma 7.4.

Lemma 7.3 below is based on traditional heavy-tailed techniques based on Chernoff's inequality for truncated random variables, such as those used in [9] and [30], to name some references. The reason why we cannot simply use existing results is our need to guarantee that the bounds do not depend on the distribution of the summands, which will be key when we apply them to $W_{n}$. Hence, special care goes into accounting for the constants explicitly; see [20] for the proof. The corollary that we obtain from this lemma will be used in the proof of Lemma 7.4.

Lemma 7.3. Suppose that $Y_{1}, Y_{2}, \ldots$ are nonnegative i.i.d. random variables with the same distribution as $Y$, where $\mathrm{E}\left[Y^{\beta}\right]<\infty$ for some $\beta>0$. Fix $0<\epsilon<1$. Then

1. for $0<\beta<1,1 \leq k \leq x^{\beta} / \mathrm{E}\left[Y^{\beta}\right]$, and $x \geq \exp \left[(K \mathrm{e})^{1 /(1-\beta)}\right]$,

$$
\begin{aligned}
& \mathrm{P}\left(\sum_{i=1}^{k} Y_{i}>x, \max _{1 \leq i \leq k} Y_{i} \leq \frac{x}{\log x}\right) \\
& \quad \leq \exp \left[-(1-\beta)(\log x)(\log \log x)\left(1-\frac{\log (\mathrm{e} K)}{(1-\beta) \log \log x}\right)\right],
\end{aligned}
$$

2. for $\beta>1,1 \leq k \leq(1-\epsilon) x /\left(\mathrm{E}[Y] \vee \mathrm{E}\left[Y^{\beta}\right]\right)$, and $x \geq \mathrm{e} \vee(K \mathrm{e} / \epsilon)^{2 /(\beta-1)}$,

$$
\begin{aligned}
& \mathrm{P}\left(\sum_{i=1}^{k} Y_{i}>x, \max _{1 \leq i \leq k} Y_{i} \leq \frac{x}{\log x}\right) \\
& \quad \leq \exp \left[-\epsilon(\beta-1)(\log x)^{2}\left(1-\frac{\log \log x}{\log x}-\frac{\log (K \mathrm{e} / \epsilon)}{(\beta-1) \log x}\right)+\mathrm{e}^{5}(\beta-1)^{2}\right],
\end{aligned}
$$

where $K=K(\beta)>1$ is a constant that does not depend on $\epsilon, k$, or the distribution of $Y$.

As an immediate corollary to the preceding lemma, we obtain the following result. 
Corollary 7.1. Suppose that $Y_{1}, Y_{2}, \ldots$ are nonnegative i.i.d. random variables with the same distribution as $Y$, where $\mathrm{E}\left[Y^{\beta}\right]<\infty$ for some $\beta>0$. Then, for any $\kappa>0$, there exists a constant $x_{0}>0$ that does not depend on the distribution of $Y$ such that

$$
\sup _{1 \leq k \leq m_{\beta}(x)} \mathrm{P}\left(\sum_{i=1}^{k} Y_{i}>x, \max _{1 \leq i \leq k} Y_{i} \leq \frac{x}{\log x}\right) \leq x^{-\kappa}
$$

for all $x \geq x_{0}$, where

$$
m_{\beta}(x)= \begin{cases}\frac{x^{\beta}}{\mathrm{E}\left[Y^{\beta}\right]}, & 0<\beta<1, \\ \frac{(1-\epsilon) x}{\mathrm{E}[Y] \vee \mathrm{E}\left[Y^{\beta}\right]}, & \beta>1,0<\epsilon<1 .\end{cases}
$$

Lemma 7.4 below gives a bound for the distribution of $W_{n+1}$ in terms of that of $W_{n}$. This lemma can also be used to prove the corresponding uniform bound for $W_{n}$ in the case when $Q$ dominates recursion (1.1). In the statement of the lemma we assume that $1 / L(x)$ is locally bounded on $[1, \infty)$.

Lemma 7.4. Suppose that $\mathrm{P}(N>x) \leq x^{-\alpha} L(x)$, with $\alpha>1$ and $L(\cdot)$ slowly varying, and that $\mathrm{E}[N] \max \left\{\mathrm{E}\left[C^{\alpha}\right], \mathrm{E}[C]\right\}<\eta<1$. Then, for any $c>0,0<\epsilon<1$, and $0<\delta<1 \wedge(\alpha-1) / 2$, there exist constants $K=K(\delta, \epsilon, c, \eta)>0$ and $x_{0}=x_{0}(\delta, \epsilon, c, \eta)>0$, which do not depend on $n$, such that, for all $1 \leq n \leq c \log x /|\log \eta|$ and all $x \geq x_{0}$,

$$
\mathrm{P}\left(W_{n+1}>x\right) \leq K \eta^{(2 \wedge(\alpha-\delta)) n} x^{-\alpha} L(x)+\mathrm{E}[N] \mathrm{P}\left(C W_{n}>(1-\epsilon) x\right),
$$

where $C$ and $W_{n}$ are independent.

Remark. Note that the condition $\mathrm{E}[N] \max \left\{\mathrm{E}\left[C^{\alpha}\right], \mathrm{E}[C]\right\}<1$ is natural since it is needed for the finiteness of $\mathrm{E}\left[R^{\beta}\right]$ for any $\beta<\alpha$. It is also in agreement with Lemma 5.1 in the sense that it is a necessary condition for the convergence (as $n \rightarrow \infty)$ of the sum appearing in (5.1). The choice of $\eta$ is also suggested by the fact that, for $\beta<\alpha$, we can obtain a weaker uniform bound by applying the moment estimate on $\mathrm{E}\left[W_{n}^{\beta}\right]$ from Lemma 3.2, i.e. $\mathrm{P}\left(W_{n}>x\right) \leq \mathrm{E}\left[W_{n}^{\beta}\right] x^{-\beta} \leq K_{\beta}\left(\mathrm{E}[N] \max \left\{\mathrm{E}[C], \mathrm{E}\left[C^{\beta}\right]\right\}\right)^{n} x^{-\beta}$.

Before presenting the proof, we would like to emphasize that special care goes into making sure that $K$ and $x_{0}$ in the statement of the lemma do not depend on $n$. This is important since Lemma 7.4 will be applied iteratively in the proof of Proposition 5.1, where we do not want $K$ and $x_{0}$ to grow from one iteration to the next.

Proof of Lemma 7.4. By convexity of $f(\theta)=\mathrm{E}\left[C^{\theta}\right], \max \left\{\mathrm{E}\left[C^{\alpha}\right], \mathrm{E}[C]\right\} \geq \max \left\{\mathrm{E}\left[C^{\alpha-\delta}\right]\right.$, $\mathrm{E}[C]\}$, implying that

$$
\varepsilon:=\frac{\eta}{\mathrm{E}[N] \max \left\{\mathrm{E}\left[C^{\alpha-\delta}\right], \mathrm{E}[C]\right\}}-1>0 .
$$

Next, recall that $W_{n+1} \stackrel{\mathrm{D}}{=} \sum_{i=1}^{N} C_{i} W_{n, i}$, where the $W_{n, i}$ are i.i.d. copies of $W_{n}$, and let $Y \stackrel{\mathrm{D}}{=} Y_{i}=$ $C_{i} W_{n, i}$ and $\beta=\alpha-\delta>1$. Note that, by Lemma 3.2, there exists a constant $K_{1}>0$ (that does not depend on $n$ ) such that

$$
\mathrm{E}\left[Y^{\beta}\right]=\mathrm{E}\left[C^{\beta}\right] \mathrm{E}\left[W_{n}^{\beta}\right] \leq K_{1}\left(\mathrm{E}[N] \max \left\{\mathrm{E}\left[C^{\alpha-\delta}\right], \mathrm{E}[C]\right\}\right)^{n}=K_{1}(1+\varepsilon)^{-n} \eta^{n},
$$

where the last equality comes from the definition of $\varepsilon$. Since $\mathrm{E}[Y]=\mathrm{E}[Q](\mathrm{E}[N] \mathrm{E}[C])^{n} \leq$ $\mathrm{E}[Q]\left(\mathrm{E}[N] \max \left\{\mathrm{E}\left[C^{\alpha-\delta}\right], \mathrm{E}[C]\right\}\right)^{n}$, then

$$
\mathrm{E}\left[Y^{\beta}\right] \vee \mathrm{E}[Y] \leq K_{2}(1+\varepsilon)^{-n} \eta^{n}
$$


for some constant $K_{2}>0$ that does not depend on $n$. With the intent of applying Corollary 7.1, we define

$$
y:=\epsilon x \quad \text { and } \quad m_{\beta}(x):=\left\lfloor\frac{\epsilon^{2} x}{\mathrm{E}\left[Y^{\beta}\right] \vee \mathrm{E}[Y]}\right\rfloor .
$$

Let $M_{k}^{(i)}$ be the $i$ th order statistic of $\left\{Y_{1}, \ldots, Y_{k}\right\}$, with $M_{k}^{(k)}$ being the largest. Then

$$
\begin{aligned}
\mathrm{P}\left(W_{n+1}>x\right)= & \mathrm{P}\left(\sum_{i=1}^{N} Y_{i}>x\right) \\
\leq & \mathrm{P}\left(\sum_{i=1}^{N} Y_{i}>x, N \leq m_{\beta}(x)\right)+\mathrm{P}\left(N>m_{\beta}(x)\right) \\
\leq & \mathrm{P}\left(\sum_{i=1}^{N} Y_{i}>x, M_{N}^{(N)} \leq(1-\epsilon) x, N \leq m_{\beta}(x)\right) \\
& +\mathrm{P}\left(M_{N}^{(N)}>(1-\epsilon) x, N \leq m_{\beta}(x)\right)+\mathrm{P}\left(N>m_{\beta}(x)\right) \\
\leq & \mathrm{P}\left(\sum_{i=1}^{N} Y_{i}>x, M_{N}^{(N)} \leq(1-\epsilon) x, M_{N}^{(N-1)} \leq \frac{y}{\log y}, N \leq m_{\beta}(x)\right) \\
& +\mathrm{P}\left(M_{N}^{(N-1)}>\frac{y}{\log y}, N \leq m_{\beta}(x)\right) \\
& +\mathrm{P}\left(M_{N}^{(N)}>(1-\epsilon) x, N \leq m_{\beta}(x)\right)+\mathrm{P}\left(N>m_{\beta}(x)\right) .
\end{aligned}
$$

Note that the term in (7.5) can be bounded as follows:

$$
\begin{aligned}
& \mathrm{P}\left(\sum_{i=1}^{N} Y_{i}>x, M_{N}^{(N)} \leq(1-\epsilon) x, M_{N}^{(N-1)} \leq \frac{y}{\log y}, N \leq m_{\beta}(x)\right) \\
& \quad \leq \mathrm{P}\left(\sum_{i=1}^{N} Y_{i}-M_{N}^{(N)}>y, M_{N}^{(N-1)} \leq \frac{y}{\log y}, N \leq m_{\beta}(x)\right) \\
& \quad \leq \mathrm{P}\left(\sum_{i=1}^{N} Y_{i}>y, M_{N}^{(N)} \leq \frac{y}{\log y}, N \leq m_{\beta}(x)\right) \\
& \quad \leq \mathrm{P}\left(\sum_{i=1}^{m_{\beta}(x)} Y_{i}>y, \max _{1 \leq i<m_{\beta}(x)} Y_{i} \leq \frac{y}{\log y}\right) .
\end{aligned}
$$

Fix $v=\alpha+\delta+c(\alpha-\delta)$. Then, by Corollary 7.1, there exists a constant $x_{1} \geq \mathrm{e}$, which does not depend on the distribution of $Y$ (and, therefore, does not depend on $n$ ), such that

$$
\begin{aligned}
\mathrm{P}\left(\sum_{i=1}^{m_{\beta}(x)} Y_{i}>y, \max _{1 \leq i<m_{\beta}(x)} Y_{i} \leq \frac{y}{\log y}\right) & \leq y^{-v} \\
& =\epsilon^{-v} \eta^{c(\alpha-\delta) \log x /|\log \eta|} x^{-\alpha-\delta} \\
& \leq \epsilon^{-v} \eta^{(\alpha-\delta) n} x^{-\alpha-\delta}
\end{aligned}
$$




$$
\begin{aligned}
& =\epsilon^{-v} \frac{x^{-\delta}}{L(x)} \eta^{\beta n} x^{-\alpha} L(x) \\
& \leq \epsilon^{-v} \sup _{t \geq 1} \frac{t^{-\delta}}{L(t)} \eta^{\beta n} x^{-\alpha} L(x)
\end{aligned}
$$

for all $y \geq x_{1}$, where the second inequality follows from the assumption that $n \leq$ $c \log x /|\log \eta|$, and in the second equality we used the definition $\beta=\alpha-\delta$. To bound (7.6), we condition on $N$ :

$$
\begin{aligned}
\mathrm{P}\left(M_{N}^{(N-1)}>\frac{y}{\log y}, N \leq m_{\beta}(x)\right) & =\sum_{k=1}^{m_{\beta}(x)} \mathrm{P}\left(M_{k}^{(k-1)}>\frac{y}{\log y}\right) \mathrm{P}(N=k) \\
& \leq \sum_{k=1}^{m_{\beta}(x)}\left(\begin{array}{l}
k \\
2
\end{array}\right) \mathrm{P}\left(Y>\frac{y}{\log y}\right)^{2} \mathrm{P}(N=k) \\
& \leq \mathrm{E}\left[N^{2} \mathbf{1}_{\left.\left\{N \leq m_{\beta}(x)\right\}\right]} \mathrm{P}\left(Y>\frac{y}{\log y}\right)^{2}\right. \\
& \leq \mathrm{E}\left[N^{2 \wedge \beta}\right] m_{\beta}(x)^{(2-\beta)^{+}} \mathrm{P}\left(Y>\frac{y}{\log y}\right)^{2},
\end{aligned}
$$

where in the last inequality we used $N \leq m_{\beta}(x)$ in case $N$ does not have a second moment. Now, by Markov's inequality and the definition of $m_{\beta}(x)$,

$$
\begin{aligned}
m_{\beta}(x)^{(2-\beta)^{+}} \mathrm{P}\left(Y>\frac{y}{\log y}\right)^{2} & \leq m_{\beta}(x)^{(2-\beta)^{+}}\left(\frac{\mathrm{E}\left[Y^{\beta}\right](\log y)^{\beta}}{y^{\beta}}\right)^{2} \\
& \leq\left(\frac{\mathrm{E}\left[Y^{\beta}\right]}{\mathrm{E}\left[Y^{\beta}\right] \vee \mathrm{E}[Y]}\right)^{(2-\beta)^{+}} \frac{\epsilon^{(2-\beta)^{+}} \mathrm{E}\left[Y^{\beta}\right]^{2 \wedge \beta}(\log y)^{2 \beta}}{y^{2 \beta \wedge(3 \beta-2)}} \\
& \leq \frac{\epsilon^{(2-\beta)^{+}} \mathrm{E}\left[Y^{\beta}\right]^{2 \wedge \beta}(\log y)^{2 \beta}}{y^{2 \beta \wedge(3 \beta-2)}} \\
& \leq \frac{\epsilon^{(2-\beta)^{+}}\left(K_{1}(1+\varepsilon)^{-n} \eta^{n}\right)^{2 \wedge \beta}(\log y)^{2 \beta}}{y^{2 \beta \wedge(3 \beta-2)}} \quad \text { by (7.3)). }
\end{aligned}
$$

Our choice of $\delta$ guarantees that $2 \beta \wedge(3 \beta-2)>\alpha+\delta$ and $\beta=\alpha-\delta>1$, and, therefore,

$$
\begin{aligned}
\mathrm{P}\left(M_{N}^{(N-1)}>\frac{y}{\log y}, N \leq m_{\beta}(x)\right) & \leq K_{3} \frac{\eta^{(2 \wedge \beta) n}}{(1+\varepsilon)^{(2 \wedge \beta) n}} x^{-\alpha-\delta} \\
& \leq K_{3} \frac{x^{-\delta}}{L(x)} \eta^{(2 \wedge \beta) n} x^{-\alpha} L(x) \\
& \leq K_{3} \sup _{t \geq 1} \frac{t^{-\delta}}{L(t)} \eta^{(2 \wedge \beta) n} x^{-\alpha} L(x)
\end{aligned}
$$

for all $x \geq x_{2}=\epsilon^{-1} \mathrm{e}$, where

$$
K_{3}=K_{3}(\epsilon, \delta)=\mathrm{E}\left[N^{2 \wedge \beta}\right] \epsilon^{(2-\beta)^{+}-\alpha-\delta} K_{1}^{2 \wedge \beta} \sup _{t \geq \mathrm{e}} \frac{(\log t)^{2 \beta}}{t^{2 \beta \wedge(3 \beta-2)-\alpha-\delta}} .
$$


To bound the second term in (7.7), we first note that, by Potter's theorem (see Theorem 1.5.6(ii) of [8, p. 25]), there exists a constant $x_{3}=x_{3}(\varepsilon, \delta)$ such that, for all $x \geq x_{3}$,

$$
\begin{aligned}
\mathrm{P}\left(N>m_{\beta}(x)\right) & \leq \frac{\left(m_{\beta}(x)\right)^{-\alpha} L\left(m_{\beta}(x)\right)}{x^{-\alpha} L(x)} x^{-\alpha} L(x) \\
& \leq(1+\varepsilon) \max \left\{\left(\frac{m_{\beta}(x)}{x}\right)^{-\alpha+\delta},\left(\frac{m_{\beta}(x)}{x}\right)^{-\alpha-\delta}\right\} x^{-\alpha} L(x) \\
& =(1+\varepsilon) \max \left\{\left(\frac{\mathrm{E}\left[Y^{\beta}\right] \vee \mathrm{E}[Y]}{\epsilon^{2}}\right)^{\alpha-\delta},\left(\frac{\mathrm{E}\left[Y^{\beta}\right] \vee \mathrm{E}[Y]}{\epsilon^{2}}\right)^{\alpha+\delta}\right\} x^{-\alpha} L(x) \\
& \leq \frac{1+\varepsilon}{\epsilon^{2(\alpha+\delta)}}\left(\mathrm{E}\left[Y^{\beta}\right] \vee \mathrm{E}[Y]\right)^{\beta} x^{-\alpha} L(x) \\
& \leq \frac{K_{2}^{\beta}}{\epsilon^{2(\alpha+\delta)}} \frac{\eta^{\beta n}}{(1+\varepsilon)^{\beta n-1}} x^{-\alpha} L(x) \quad(\text { by (7.4)) } \\
& \leq K_{4} \eta^{\beta n} x^{-\alpha} L(x) .
\end{aligned}
$$

Finally, for the first term in (7.7),

$$
\begin{aligned}
\mathrm{P}\left(M_{N}^{(N)}>(1-\epsilon) x, N \leq m_{\beta}(x)\right) & \leq \mathrm{P}\left(M_{N}^{(N)}>(1-\epsilon) x\right) \\
& \leq \mathrm{E}[N] \mathrm{P}(Y>(1-\epsilon) x) .
\end{aligned}
$$

Combining the preceding bounds for (7.5)-(7.7), and setting $x_{0}=\max \left\{x_{1}, x_{2}, x_{3}\right\}$ and $K=$ $\left(\epsilon^{-v}+K_{3}\right) \sup _{t \geq 1} t^{-\delta} / L(t)+K_{4}$ completes the proof.

Finally, we give the proof of Proposition 5.1, the main technical contribution of Section 5.

Proof of Proposition 5.1. Note that it is enough to prove the proposition for all $x \geq x_{0}$ for some $x_{0}=x_{0}(\eta, v)>1$, since, for all $1 \leq x \leq x_{0}$ and $n \geq 1$,

$$
\begin{aligned}
\mathrm{P}\left(W_{n}>x\right) & =\frac{\mathrm{P}\left(W_{n}>x\right)}{\eta^{n} \mathrm{P}(N>x)} \eta^{n} \mathrm{P}(N>x) \\
& \leq \frac{\mathrm{E}[Q](\mathrm{E}[N] \mathrm{E}[C])^{n} x^{-1}}{\eta^{n} \mathrm{P}(N>x)} \eta^{n} \mathrm{P}(N>x) \quad \text { (by Markov's inequality) } \\
& \leq \sup _{1 \leq t \leq x_{0}} \frac{\mathrm{E}[Q]}{t \mathrm{P}(N>t)} \eta^{n} \mathrm{P}(N>x) .
\end{aligned}
$$

Next, choose $0<\epsilon<1$ such that

$$
\mathrm{E}[N] \mathrm{E}\left[C^{\alpha}\right]\left((1-\epsilon)^{-\alpha-1}+2 \epsilon\right) \leq \eta,
$$

define $c=v / 2$,

$$
\gamma=\frac{1}{|\log \eta|} \log \left(\frac{\eta}{\mathrm{E}[N] \max \left\{\mathrm{E}\left[C^{\alpha}\right], \mathrm{E}[C]\right\}}\right),
$$

and select $0<\delta<\min \{1,(\alpha-1) / 2, c \gamma\}$. Now, by Lemma 7.4, there exist constants $K_{1}$, $x_{1}>0$ (that do not depend on $n$ ) such that

$$
\mathrm{P}\left(W_{n+1}>x\right) \leq K_{1} \eta^{(2 \wedge(\alpha-\delta)) n} \mathrm{P}(N>x)+\mathrm{E}[N] \mathrm{P}\left(C W_{n}>(1-\epsilon) x\right) \text { for all } x \geq x_{1} .
$$


Hence, by defining $n_{0}=(2 \wedge(\alpha-\delta)-1)^{-1}(\log \eta)^{-1} \log \left(\epsilon \mathrm{E}[N] \mathrm{E}\left[C^{\alpha}\right]\right)$, we obtain

$$
\mathrm{P}\left(W_{n+1}>x\right) \leq K_{1} \mathrm{E}[N] \mathrm{E}\left[C^{\alpha}\right] \epsilon \eta^{n} \mathrm{P}(N>x)+\mathrm{E}[N] \mathrm{P}\left(C W_{n}>(1-\epsilon) x\right)
$$

for all $n \geq n_{0}$ and all $x \geq x_{1}$.

Next, in order to derive an explicit bound for $\mathrm{P}\left(W_{n}>x\right)$, we need the two estimates, (7.10) and (7.11), below. In this regard, choose $x_{0} \geq 1 \vee x_{1}$ such that

$$
\mathrm{P}(C N>(1-\epsilon) x) \leq \mathrm{E}\left[C^{\alpha}\right](1-\epsilon)^{-\alpha-1} \mathrm{P}(N>x)
$$

for all $x \geq x_{0}$. This is possible since, by Lemma 4.2 of [22], $\mathrm{P}(C N>(1-\epsilon) x) \sim \mathrm{E}\left[C^{\alpha}\right](1-$ $\epsilon)^{-\alpha} \mathrm{P}(N>x)$. Also, by Markov's inequality we have, for all $1 \leq n \leq c \log x /|\log \eta|$,

$$
\begin{aligned}
\mathrm{P}\left(C>\frac{(1-\epsilon) x}{x_{0}}\right) & \leq \mathrm{E}\left[C^{\alpha+v}\right](1-\epsilon)^{-\alpha-v} x_{0}^{\alpha+v} x^{-\alpha-v} \\
& =\frac{\mathrm{E}\left[C^{\alpha+v}\right] x_{0}^{\alpha+v}}{(1-\epsilon)^{\alpha+v} x^{v / 2} L(x)} x^{-v / 2} \mathrm{P}(N>x) \\
& \leq \frac{\mathrm{E}\left[C^{\alpha+v}\right] x_{0}^{\alpha+v}}{(1-\epsilon)^{\alpha+v} x^{\nu / 2} L(x)} \eta^{n} \mathrm{P}(N>x),
\end{aligned}
$$

where in the second inequality we used $x^{-v / 2}=x^{-c}=\eta^{c \log x /|\log \eta|} \leq \eta^{n}$. Now, define

$$
K_{2}=\max \left\{1, K_{1}, \sup _{x \geq x_{0}} \frac{\mathrm{E}\left[C^{\alpha+\nu}\right] x_{0}^{\alpha+v}}{\epsilon \mathrm{E}\left[C^{\alpha}\right](1-\epsilon)^{\alpha+v} x^{\nu / 2} L(x)}\right\} .
$$

Now we proceed to derive bounds for $\mathrm{P}\left(W_{n}>x\right)$ for different ranges of $n$. For all $1 \leq n \leq n_{0}$ and all $x \geq x_{0}$, by Lemma 5.1, there exists a constant $K_{0} \geq K_{2}$ such that

$$
\mathrm{P}\left(W_{n}>x\right) \leq K_{0} \eta^{n} \mathrm{P}(N>x) .
$$

Next, for the values $n_{0} \leq n \leq c \log x /|\log \eta|$, we proceed by induction using (7.9). To this end, suppose that (7.12) holds for some $n$ in the specified range. Then, note that, by (7.11) and the induction hypothesis (7.12), we have, for all $x \geq x_{0}$,

$$
\begin{aligned}
\mathrm{P}(C & \left.W_{n}>(1-\epsilon) x\right) \\
& \leq \mathrm{P}\left(C W_{n}>(1-\epsilon) x, C \leq \frac{(1-\epsilon) x}{x_{0}}\right)+\mathrm{P}\left(C>\frac{(1-\epsilon) x}{x_{0}}\right) \\
& \leq \int_{0}^{(1-\epsilon) x / x_{0}} \mathrm{P}\left(W_{n}>\frac{(1-\epsilon) x}{y}\right) \mathrm{P}(C \epsilon \mathrm{d} y)+K_{2} \mathrm{E}\left[C^{\alpha}\right] \epsilon \eta^{n} \mathrm{P}(N>x) \\
& \leq K_{0} \eta^{n} \int_{0}^{\infty} \mathrm{P}\left(N>\frac{(1-\epsilon) x}{y}\right) \mathrm{P}(C \in \mathrm{d} y)+K_{2} \mathrm{E}\left[C^{\alpha}\right] \epsilon \eta^{n} \mathrm{P}(N>x) \\
& =K_{0} \eta^{n} \mathrm{P}(C N>(1-\epsilon) x)+K_{2} \mathrm{E}\left[C^{\alpha}\right] \epsilon \eta^{n} \mathrm{P}(N>x) \\
& \leq K_{0} \mathrm{E}\left[C^{\alpha}\right]\left((1-\epsilon)^{-\alpha-1}+\epsilon\right) \eta^{n} \mathrm{P}(N>x),
\end{aligned}
$$

where in the last inequality we used (7.10) and $K_{0} \geq K_{2}$. Then, by replacing the preceding bound in (7.9) and using (7.8), we derive

$$
\begin{aligned}
\mathrm{P}\left(W_{n+1}>x\right) & \leq K_{0} \mathrm{E}[N] \mathrm{E}\left[C^{\alpha}\right]\left((1-\epsilon)^{-\alpha-1}+2 \epsilon\right) \eta^{n} \mathrm{P}(N>x) \\
& \leq K_{0} \eta^{n+1} \mathrm{P}(N>x)
\end{aligned}
$$

for all $x \geq x_{0}$ and all $1 \leq n \leq c \log x /|\log \eta|$. 
Finally, for $n \geq c \log x /|\log \eta|$, we follow a different approach that comes from our moment estimates for $W_{n}$. Let

$$
\varepsilon=\frac{\eta}{\mathrm{E}[N] \max \left\{\mathrm{E}\left[C^{\alpha}\right], \mathrm{E}[C]\right\}}-1>0
$$

and note that, by convexity,

$$
\mathrm{E}[N] \max \left\{\mathrm{E}\left[C^{\alpha-\delta}\right], \mathrm{E}[C]\right\} \leq \mathrm{E}[N] \max \left\{\mathrm{E}\left[C^{\alpha}\right], \mathrm{E}[C]\right\}=(1+\varepsilon)^{-1} \eta .
$$

Then, by Markov's inequality and Lemma 3.2, we have

$$
\begin{aligned}
\mathrm{P}\left(W_{n}>x\right) & \leq \mathrm{E}\left[W_{n}^{\alpha-\delta}\right] x^{-\alpha+\delta} \\
& \leq K_{\alpha-\delta}\left(\mathrm{E}[N] \max \left\{\mathrm{E}\left[C^{\alpha-\delta}\right], \mathrm{E}[C]\right\}\right)^{n} x^{-\alpha+\delta} \\
& =K_{\alpha-\delta}(1+\varepsilon)^{-n} \eta^{n} x^{-\alpha+\delta} \\
& \leq K_{\alpha-\delta} x^{-\log (1+\varepsilon) c /|\log \eta|} \eta^{n} x^{-\alpha+\delta}
\end{aligned}
$$

for all $x>0$. Note that the preceding bound,

$$
\frac{\log (1+\varepsilon)}{|\log \eta|}=\frac{1}{|\log \eta|} \log \left(\frac{\eta}{\mathrm{E}[N] \max \left\{\mathrm{E}\left[C^{\alpha}\right], \mathrm{E}[C]\right\}}\right)=\gamma,
$$

and (7.13) yield

$$
\begin{aligned}
\mathrm{P}\left(W_{n}>x\right) & \leq K_{\alpha-\delta} \eta^{n} x^{-c \gamma-\alpha+\delta} \\
& \leq K_{\alpha-\delta} \eta^{n} x^{-\alpha+\delta-c \gamma} \\
& =K_{\alpha-\delta} \eta^{n} \frac{x^{\delta-c \gamma}}{L(x)} \mathrm{P}(N>x) \\
& \leq K_{\alpha-\delta} \sup _{t \geq 1} \frac{t^{\delta-c \gamma}}{L(t)} \eta^{n} \mathrm{P}(N>x)
\end{aligned}
$$

for all $x \geq 1$; recall that $\delta<c \gamma$. Thus, setting $K=\max \left\{K_{0}, K_{\alpha-\delta} \sup _{t \geq 1} t^{\delta-c \gamma}(L(t))^{-1}\right\}$ completes the proof.

\subsection{The case when $Q$ dominates}

We end the paper with a sketch of the proof of Proposition 6.1. As mentioned before, the proofs of the other results presented in Section 6 have been omitted since they are very similar to those from Section 5 .

Sketch of the proof of Proposition 6.1. By Markov's inequality,

$$
\mathrm{P}(N>x) \leq \mathrm{E}\left[N^{\alpha+v}\right] x^{-\alpha-v}
$$

for all $x>0$. Use Lemma 7.4 to obtain

$$
\mathrm{P}\left(W_{n+1}>x\right) \leq K_{1} \mathrm{E}[N] \mathrm{E}\left[C^{\alpha}\right] \epsilon \eta^{n} \mathrm{P}(Q>x)+\mathrm{E}[N] \mathrm{P}\left(C W_{n}>(1-\epsilon) x\right)
$$

for all $n_{0} \leq n \leq \kappa \log x$ and all $x \geq x_{1}$ (for suitably chosen constants $\epsilon, n_{0}$, and $\kappa$ ). Choose $x_{0} \geq 1 \vee x_{1}$ such that

$$
\mathrm{P}(C Q>(1-\epsilon) x) \leq \mathrm{E}\left[C^{\alpha}\right](1-\epsilon)^{-\alpha-1} \mathrm{P}(Q>x) .
$$

The rest of the proof continues as in Proposition 5.1 with some modifications. 


\section{Acknowledgements}

The authors are grateful to Professor Charles Goldie for pointing out a reference, and also to an anonymous referee for his or her helpful comments.

\section{References}

[1] Alsmeyer, G. and Kuhlbusch, D. (2010). Double martingale structure and existence of $\phi$-moments for weighted branching processes. To appear in Münster J. Math.

[2] Alsmeyer, G. AND Rösler, U. (2006). A stochastic fixed point equation related to weighted branching with deterministic weights. Electron. J. Prob. 11, 27-56.

[3] Asmussen, S. (1998). Subexponential asymptotics for stochastic processes: extremal behavior, stationary distributions and first passage probabilities. Ann. Appl. Prob. 8, 354-474.

[4] Asmussen, S. (2003). Applied Probability and Queues. Springer, New York.

[5] Athreya, K. B., McDonald, D. And Ney, P. (1978). Limit theorems for semi-Markov processes and renewal theory for Markov chains. Ann. Prob. 6, 788-797.

[6] Athreya, K. B. And Ney, P. E. (2004). Branching Processes. Dover, Mineola, NY.

[7] Baltrūnas, A., Daley, D. J. And Klüppelberg, C. (2004). Tail behavior of the busy period of a GI/GI/1 queue with subexponential service times. Stoch. Process. Appl. 111, 237-258.

[8] Bingham, N. H., Goldie, C. M. And Teugels, J. L. (1987). Regular Variation. Cambridge University Press.

[9] Borovkov, A. (2000). Estimates for the distribution of sums and maxima of sums of random variables without the Cramér condition. Siberian Math J. 41, 811-848.

[10] Brandt, A. (1986). The stochastic equation $y_{n+1}=a_{n} y_{n}+b_{n}$ with stationary coefficients. Adv. Appl. Prob. 18, 211-220.

[11] Brin, S. And Page, L. (1998). The anatomy of a large-scale hypertextual Web search engine. Comput. Networks ISDN Systems 30, 107-117.

[12] Chow, Y. S. And Teicher, H. (1988). Probability Theory, 2nd edn. Springer, New York.

[13] De Meyer, A. And Teugels, J. L. (1980). On the asymptotic behaviour of the distributions of the busy period and service time in M/G/1. J. Appl. Prob. 17, 802-813.

[14] Denisov, D., Foss, S. And Korshunov, D. (2009). Asymptotics of randomly stopped sums in the presence of heavy tails. Preprint. Available at the http://arxiv.org/abs/0808.3697v3.

[15] Fill, J. A. And Janson, S. (2001). Approximating the limiting Quicksort distribution. Random Structures Algorithms 19, 376-406.

[16] Goldie, C. M. (1991). Implicit renewal theory and tails of solutions of random equations. Ann. Appl. Prob. 1, 126-166.

[17] Gyöngyi, Z., Garcia-Molina, H. and Pedersen, J. (2004). Combating Web spam with TrustRank. Tech. Rep., Stanford University.

[18] IKsanov, A. M. (2004). Elementary fixed points of the BRW smoothing transforms with infinite number of summands. Stoch. Process. Appl. 114, 27-50.

[19] Jelenković, P. R. And MomčIlović, P. (2004). Large deviations of square root insensitive random sums. Math. Operat. Res. 29, 398-406.

[20] Jelenković, P. R. and Olvera-Cravioto, M. (2009). Information ranking and power laws on trees. Preprint. Available at http://arxiv.org/abs/0905.1738.

[21] Jelenković, P. R. AND TAN, J. (2010). Modulated branching processes, origins of power laws and queueing duality. Math. Operat. Res. 35, 807-829.

[22] Jessen, A. H. and Mikosch, T. (2006). Regularly varying functions. Publ. Inst. Math. 80, 171-192.

[23] Kesten, H. (1973). Random difference equations and renewal theory for products of random matrices. Acta Math. 131, 207-248.

[24] Kleinberg, J. M. (1999). Authoritative sources in a hyperlinked environment. J. ACM 46, 604-632.

[25] Kuhlbusch, D. (2004). On weighted branching processes in random environment. Stoch. Process. Appl. 109, 113-144.

[26] Litvak, N., Scheinhardt, W. R. W. And Volkovich, Y. (2007). In-degree and PageRank: why do they follow similar power laws? Internet Math. 4, 175-198.

[27] LIU, Q. (1998). Fixed points of a generalized smoothing transformation and applications to the branching random walk. Adv. Appl. Prob. 30, 85-112.

[28] LiU, Q. (2000). On generalized multiplicative cascades. Stoch. Process. Appl. 86, 263-286.

[29] Mikosch, T. and Samorodnitsky, G. (2000). The supremum of a negative drift random walk with dependent heavy-tailed steps. Ann. Appl. Prob. 10, 1025-1064.

[30] Nagaev, S. V. (1982). On the asymptotic behavior of one-sided large deviation probabilities. Theory Prob. Appl. 26, 362-366. 
[31] RöSLER, U. (1993). The weighted branching process. In Dynamics of Complex and Irregular Systems (Bielefeld, 1991), World Science Publishing, River Edge, NJ, pp. 154-165.

[32] RöSler, U. ANd RÜSCHENDORF, L. (2001). The contraction method for recursive algorithms. Algorithmica 29, 3-33.

[33] Rösler, U., Topchi, V. A. And Vatutin, V. A. (2000). Convergence conditions for the weighted branching process. Discrete Math. Appl. 10, 5-21.

[34] Volkovich, Y. (2009). Stochastic analysis of Web page ranking. Doctoral Thesis, University of Twente.

[35] Volkovich, Y. and Litvak, N. (2010). Asymptotic analysis for personalized Web search. Adv. Appl. Prob. 42, 577-604.

[36] Volkovich, Y., Litvak, N. and Donato, D. (2007). Determining factors behind the Pagerank log-log plot. In Algorithms and Models for the Web-Graph, Springer, Berlin, pp. 108-123.

[37] Zwart, A. P. (2001). Tail asymptotics for the busy period in the GI/G/1 queue. Math. Operat. Res. 26, $485-493$. 